\title{
A laboratory-scale buried charge simulator
}

\author{
G.J. McShane, V.S. Deshpande, N.A. Fleck \\ Department of Engineering, University of Cambridge \\ Trumpington Street Cambridge, CB2 1PZ, UK.
}

\begin{abstract}
An experimental technique has been developed in order to mimic the effect of landmine-loading on materials and structures to be studied in a laboratory setting, without the need for explosives. Compressed gas is discharged beneath a sand layer, simulating the dynamic flow generated by a buried explosive. High speed photography reveals that the stages of soil motion observed during a landmine blast are replicated. The effect of soil saturation and the depth of the sand layer on sand motion are evaluated. Two series of experiments have been performed with the buried charge simulator to characterise subsequeny impact of the sand. First, the time variation in pressure and impulse during sand impact on a stationary target is evaluated using a Kolsky bar apparatus. It is found that the pressure pulse imparted to the Kolsky bar consists of two phases: an initial transient phase of high pressure (attributed to wave propagation effects in the impacting sand), followed by a lower pressure phase of longer duration (due to GRAHAM, DO YOU MEAN LATERAL OR AXIAL FLOW? flow of the sand against the Kolsky bar). Both phases make a significant contribution to the total imparted impulse. It is found that wet sand exerts higher peak pressures and imparts a larger total impulse than dry sand. The level of imparted impulse is determined as a function of sand depths, and of stand-off distance between the sand and the impacted end of the the Kolsky bar. The second study uses a vertical impulse pendulum to measure the momentum imparted by sand impact to a target which is free to move vertically. The effect of target mass upon imparted momentum is investigated. It is concluded that the laboratoryscale sand impact apparatus is a flexible tool for investigating the interactions between structures and dynamic sand flows.
\end{abstract}




\section{Introduction}

Buried explosives pose a serious threat to both military and civilian vehicles in many conflict areas (Braid, 2001). Effective protection can be provided to larger vehicles by increasing the weight of armour and the ground clearance (Lester, 1996). In contrast, smaller, lighter vehicles remain vulnerable. Optimising light-weight materials for landmine resistance presents a number of unique challenges. First, the dynamic loading induced by a high velocity soil flow must be characterised. Important parameters include the size and shape of the explosive charge, the properties of the soil, the depth of burial of the explosive, and the stand-off between the structure and the charge (Tremblay, 1998). Second, the coupling between the soil impact loading and the deformation of the structure must be understood, i.e. the fluidstructure interaction (FSI). FSI effects have been studied for blasts in air (Kambouchev et al., 2006) and water (Fleck and Deshpande, 2004, Liang et al., 2008, Wadley et al., 2008). For underwater blasts in particular, it has been found that sandwich panels with carefully specified core properties can outperform monolithic plates of the same mass by exploiting FSI effects. The significance of such effects during a landmine blast remains the subject of ongoing research. The objective of the present work is to develop a laboratory scale experimental tool to allow the systematic investigation of structural response to soil impact.

The dynamic soil flow resulting from the detonation of a buried explosive has been investigated experimentally by Bergeron et al. (1998) and Braid et al. (2001), using small anti-personnel landmines at a variety of depths of burial in standard building sand. Three distinct phases are identified (see also Deshpande et al. (2009) for a detailed discussion). In the first phase, detonation of the solid explosive results in its rapid conversion into high pressure gaseous products. A compressive wave propagates into the soil, dissipating some energy in plastic deformation of the soil and (close to the charge) fragmentation of soil grains. The second phase begins with the reflection of this compressive wave from the free surface of the soil. A dome-like soil cap expands, accelerated by the detonation products contained behind. In the third phase, the cohesion of the soil cap is lost, and a loose but high velocity flow of soil develops. The ejected soil evolves into the shape of an expanding inverted cone, the included angle reducing as the depth of burial is increased.

Recent numerical work has advanced understanding of the complex interactions occurring during landmine detonation and the resulting soil loading of structures (Grujicic et al. 2006, 2008, Deshpande et al. 2009). Repeatable, instrumented 
experiments are essential for supporting these predictive modelling efforts. At present, only a limited number of experimental studies have been reported. Empirical relationships for the total impulse imparted to heavy blast deflectors have been derived from instrumented landmine experiments (Westine et al., 1985, Tremblay, 1998). These reveal that the imparted impulse depends on the stand-off (the vertical distance above the soil surface), the depth of burial (the soil over-burden) and the horizontal distance from the landmine centre TO WHAT?. However, the accurate extrapolation of these empirical models to more extreme loadining conditions is problematic.

Reduced-scale experiments are attractive for detailed parametric studies. In addition to increased safety and reduced cost, a reduced blast energy allows for a greater quantity and quality of instrumentation. Hlady (2004) employs $0.025 \mathrm{~kg}$ buried charges in conjunction with a vertical impulse pendulum to investigate the influence of soil parameters upno the blast event. Increasing the water saturation is shown to increase the momentum imparted to the target plate, but only if a threshold moisture content is exceeded. In a joint study, Taylor et al. (2005) and Fourney et al. (2005) consider the scalability of buried charge experiments. Vertical impulse pendulums were used to measure the impulse imparted by explosive charges shallow buried in fully saturated wet sand. Comparing model-scale tests (using a few grams of TNT) with full-scale experiments (with charges up to $4.5 \mathrm{~kg}$ ), a linear scaling of total imparted impulse with charge mass was suggested. The study also reveals the influence of target area and target mass on the total imparted impulse. The importance of plate area may be related to the spatial distribution of the sand flow. A dependence on plate mass indicates that FSI effects may be significant for sand impacts. Neuberger et al. (2007a,b) examined the deformation of clamped plates loaded by spherical charges either flush buried in dry sand (i.e. zero sand overburden) or in air alone. They find that the presence of the sand increases the deformation of the target plate. A scaling law based on the cube root of the charge mass ratio is shown to provide reasonable predictions of plate deformations for the air blast and buried charge cases.

In the present study, we consider an alternative approach to reduced scale sand impact experiments. Experimental studies have in the past overcome the difficulties inherent in explosive-based tests by developing 'laboratory-scale' blast simulators. Radford et al. (2005) used the impact of aluminium foam cylinders to generate dynamic shock loading. Deshpande et al. (2006) and Espinosa et al. (2006) replicated the essential features of an underwater explosion in laboratory conditions without the need for 
explosives. While not all aspects of the full scale loading event are replicated accurately, these methods have provided valuable insights into structural response to blast. We adopt this approach in the present work. A laboratory-scale buried charge simulator (BCS) has been developed which uses the rapid discharge of compressed nitrogen gas to generate a flow of sand in a manner representative of a buried explosive, albeit at significantly reduced blast energies. Sieved silica sand with a narrow range of particle sizes and carefully controlled saturation and initial packing density is used as a repeatable soil surrogate suitable for laboratory tests. The technique is amenable to instrumentation: high speed photography and dynamic pressure and impulse measurements are used to characterise the sand blast. The effects of stand-off and depth of burial upon imparted momentum are investigated.

The paper is structured as follows. In Section 2, the laboratory-scale BCS is described. The key components and their assembly are outlined, and the procedure for discharging the apparatus is given. In Section 3 we present measurements of the sand flow generated by the BCS, and make a comparison with published observations of the discharge of buried explosives. The remainder of the paper presents two series of experiments which aim to characterise the loading imparted by the sand impact. In Section 4, a Kolsky bar is used to obtain dynamic measurements of the pressure transient and impulse exerted by the sand flow onto a stationary target. The effect of sand depth, water saturation and stand-off distance are considered. In Section 5 a vertical impulse pendulum is used to assess the total momentum imparted by the sand flow to a target which is free to move vertically. Selected values are chosen for the pendulum mass to evaluate the magnitude of fluid-structure interaction effects.

\section{Description of the buried charge simulator}

The BCS is shown in cross-section in Fig. 1. The main body consists of two pressure vessels, marked 'large chamber' and 'small chamber' in Fig. 1. These are thickwalled circular cylinders with external diameter $135 \mathrm{~mm}$ and internal diameter 28 $\mathrm{mm}$, fabricated from 316L stainless steel. The large chamber has height $80 \mathrm{~mm}$, and acts as the main accumulator for storing a charge of compressed nitrogen gas. The small chamber has height $20 \mathrm{~mm}$, and is used for discharging the device in a controlled manner, as detailed subsequently. Each chamber is connected to a hose of internal diameter $6 \mathrm{~mm}$, through which nitrogen gas is supplied and evacuated (these are marked 'gas inlet 1' and 'gas inlet 2' in Fig. 1 for the small and large chambers, respectively). Piezoelectric pressure sensors are fitted to both inlets to monitor the pressure in the two chambers. The pressure chambers are separated by a circular 
metallic shim, and a clamping ring secures a second identical shim to close the top of the small chamber. The thickness and material choice for these shims controls the triggering of the device, as described below. A circular stainless steel plate of thickness $25 \mathrm{~mm}$ seals and supports the bottom of the large chamber. Rubber O-rings recessed into circular grooves seal the interfaces between each stage, as shown in Fig. 1. A circular stainless steel tray of diameter $300 \mathrm{~mm}$ mounted on top of the clamping ring contains the sand. Various depths of tray $(D)$ are used, as required for each experiment. The compressed gas is discharged through a $28 \mathrm{~mm}$ diameter circular hole at the top of the small chamber. This hole is initially sealed with a layer of aluminium foil adhered to the underside of the tray (shown as a dashed line in Fig. 1). The BCS is clamped together by six $10 \mathrm{~mm}$ diameter high tensile steel bolts arranged on a pitch circle of diameter $102 \mathrm{~mm}$.

\subsection{Operation}

The sand flow is generated by the rapid discharge of a controlled quantity of compressed nitrogen gas vertically beneath the sand layer. The procedure for discharging the BCS is as follows:

(i) Pressure vessel assembly. First, the stages of the apparatus (pressure chambers and sand tray) are assembled and bolted together, as outlined in the previous section. The metal shims separating the pressure vessels are selected to have a rupture pressure of approximately $3 p_{0} / 4$, where $p_{0}$ is the intended fill pressure of the large chamber. The sand tray is then filled to the required depth. Careful preparation of the sand layer is necessary at this stage to ensure repeatability, as described subsequently.

(ii) Pressurisation. Compressed nitrogen is first let into the small chamber through gas inlet 1 (Fig. 1), until a pressure of $p_{0} / 2$ is attained. The large chamber is then filled via gas inlet 2 to the target pressure $p_{0}$. This double chamber arrangement maintains a differential pressure across each metal shim which is below its rupture pressure $\left(3 p_{0} / 4\right)$. Solenoid valves are fitted to gas inlets 1 and 2 near to the pressure vessels. These are closed, isolating a volume $V_{0}$ of compressed gas in the large chamber, and thus controlling the stored energy.

(iii) Discharge. To trigger the gas release, a solenoid valve connected to gas inlet 1 is opened which causes the gas in the small chamber to be rapidly expelled to the atmosphere. The differential pressure across the metallic shim containing the gas in the large chamber then exceeds its rupture pressure. The two shims fail sequentially, 
and the charge of compressed gas is released. It escapes vertically beneath the sand layer, generating the dynamic flow of sand.

The energy $\left(E_{0}\right)$ available to accelerate the sand is governed by the volume $V_{0}$ and pressure $p_{0}$ of the compressed gas in the large chamber. This energy may be estimated by the work done in isentropic expansion of an ideal gas to atmospheric pressure $p_{a}$ (Tabor, 1969):

$$
E_{0}=\frac{p_{0} V_{0}}{\gamma-1}\left[1-\left(\frac{p_{a}}{p_{0}}\right)^{\frac{\gamma-1}{\gamma}}\right]
$$

The constant $\gamma$ is the specific heat capacity ratio. For all experiments reported here, we use an initial pressure $p_{0}=3 \mathrm{MPa}$. (Copper shim of thickness $0.127 \mathrm{~mm}$ in the half-hard condition was selected to separate the pressure chambers, having the correct rupture pressure). Taking $\gamma=1.4$, and using the reservoir volume $V_{0}=58 \times 10^{3} \mathrm{~mm}^{3}$, the energy $E_{0}=270 \mathrm{~J}$. TNT has a specific internal energy of $4.5 \mathrm{MJkg}^{-1}$, and therefore the energy release of the apparatus is approximately equivalent to $60 \times 10^{-6} \mathrm{~kg}$ of explosive.

\subsection{Specification and preparation of the experimental soil}

Natural soil is a complex material, with significant variability in microstructure and properties (Bolton, 1979). The list of parameters influencing its mechanical behaviour in the dense state is long, and includes: (i) particle size, (ii) particle size distribution, (iii) particle shape, (iv) packing density (the volume fraction of solid), (v) the saturation (the fraction of the inter-particle voids filled by liquid) and (vi) the constituent minerals. In the present work, in order to control the number of variables and improve repeatability we employ a model soil consisting of sieved natural uncrushed silica sand with a well controlled particle size distribution. A nominal particle size range of $150-300 \mu \mathrm{m}$ was used, with sieve results indicating that $90 \%$ falls within this range. The sand is supplied washed and dried, with a tightly controlled initial moisture content of less than $0.1 \%$ by mass, and is stored to prevent further uptake of moisture.

Two sand conditions are considered in the experiments reported here: dry sand and fully saturated wet sand. Dry sand tests are prepared as follows. The circular sand tray is loosly filled with sand (Fig. 1), and the sand is then compacted by lightly tapping a plate placed on top of the sand pile. A straight edge is then drawn across the sand tray to give a uniform depth. By compacting the sand, an initial density of $\rho_{D}=1630 \mathrm{kgm}^{-3}$ is obtained with excellent repeatability. The relative density of the 
dry sand obtained by this method $\bar{\rho}=0.60$, is slightly below the value for dense random packing of uniformly sized spheres $(\bar{\rho}=0.64)$. Wet sand specimens are initially prepared in an identical manner. A measured quantity of water is then added to the compacted sand layer to achieve full saturation, i.e. all inter-particle voids are filled with water. The water is added slowly with a dropper at points near to the tray edge, and redistributes uniformly through the sand layer by capillary action, and without perturbation of the packing density. The measured density of the wet sand is $\rho_{W}=2020 \mathrm{kgm}^{-3}$.

\section{The sand discharge}

In this section we investigate the motion of the sand flow generated by the apparatus, to allow comparison with published results for buried charges. In all cases, the compressed nitrogen in the large chamber has an initial pressure $p_{0}=3 \mathrm{MPa}$. The apparatus is discharged as outlined above, and the resulting motion of the sand is recorded using high speed photography (a Vision Research Phantom V12 camera was used at a frame rate of 6600 frames per second, with a $20 \mu$ s exposure time). The high speed images were analysed to obtain the position of the top surface of the sand at selected times.

\subsection{Dry sand}

Consider first the case of dry sand, with two layer depths: $D=15 \mathrm{~mm}$ and $D=30 \mathrm{~mm}$ (Fig. 1). The results of the high speed photography are plotted in Fig. 2: the contours show the position of the top surface of the sand at the times indicated. Introduce the Cartesian co-ordinate system $(x, y, z)$, aligned with the cylindrical axis of the BCS. Snap-shots of the location of the sand-front on the $y=0$ plane are shown in Fig. 2. Time $t=0$ corresponds to the first frame in which motion of the sand surface was observed. Selected frames from the corresponding sequence of high speed pictures are included adjacent to the contour plot, for comparison. For scale, note that the diameter of the circular sand tray is $300 \mathrm{~mm}$ in all cases.

The sand flow qualitatively matches that of a buried explosive, as reported by Bergeron et al. (1998) and Braid et al. (2001). Initially, there is a 'bubble expansion' phase, phase two in the terminology of Bergeron et al. (1998): refer to the image at $t$ $=0.45 \mathrm{~ms}$ in Fig. 2a, and $t=0.90 \mathrm{~ms}$ in Fig. $2 \mathrm{~b}$. The diameter of the 'bubble' increases with time. Finally, the bubble evolves into a diffuse spray of sand which develops into a radially expanding inverted cone: refer to the image at $t=2.3 \mathrm{~ms}$ in Fig. 2a, and $t=4.5 \mathrm{~ms}$ in Fig. 2b. This corresponds to phase three of Bergeron et al. 
(1998). As observed in the results of these authors, the included angle of this cone reduces as the depth of the sand layer is increased: compare Figs. 2(a) and 2(b). Note that the final diameter of ejected material is also larger for the $D=30 \mathrm{~mm}$ case.

For the two dry sand cases, $D=15 \mathrm{~mm}$ and $D=30 \mathrm{~mm}$, the velocity $v$ of the top surface of the sand at $x=y=0$ was calculated by comparing successive frames of the high speed photography. The velocity is plotted in Fig. 3 as a function of the current height $z$ of the sandfront along the pole $(x=y=0)$. We have chosen to use height $z$ rather than time $t$ to characterise the evolution of velocity, as we shall subsequently be measureing the pressure transient at a particular stand-off. During phase two (bubble expansion) the sand layer is continually accelerated by the gas expansion beneath it. This lasts until the sand front has reached a stand-off of around $2 D$. During the transition to phase three, the velocity of the sand front reaches a plateau. Increasing the depth of sand reduces the sand front velocity.

\subsection{Wet sand}

High speed images of the motion of fully saturated wet sand with depth $D=15 \mathrm{~mm}$ are shown in Fig.4. Again, the times shown are relative to the first frame in which sand motion was observed. The phases of motion are seen to be the same for the wet and dry cases. Initially there is a bubble expansion, followed by the development into an inverted cone which expands radially. For wet sand however, water vapour is ejected from the sand early in its motion. The onset of this process can be seen in Fig. 4a. In Fig. 4b the water vapour cloud is fully developed. The water vapour travels faster than the sand front, obscuring it from the high speed images (Fig. 4b). For the wet sand case with depth $D=15 \mathrm{~mm}$, the velocity $v$ of the top surface of the sand at $r=0$ (on the centre-line) is plotted in Figure 3 as open symbols. Results are given only up to the point at which the ejected water vapour obscures the sand front. The increased density of the wet sand results in a slightly slower sandfront than that of dry sand.

\section{Dynamic pressure and impulse measurements}

It is common practice in experimental investigations of landmine detonations to use a vertical impulse pendulum to measure the total imparted impulse (see for example Taylor et al., 2005). However, the time history of the pressure loading during the sand impact event is also important when developing lightweight blast resistant structures: large peak pressures may result in local perforation of the structure. We now employ 
the laboratory scale sand impact apparatus to investigate the time variation in pressure and impulse imparted by the sand impact.

\subsection{Experimental procedure}

Dynamic pressure measurements were obtained using a standard strain-gauged Kolsky bar (Dharan and Hauser, 1970), as follows. A circular cylindrical aluminium alloy bar (2017-T4), ofdiameter $12.6 \mathrm{~mm}$ and length $2.2 \mathrm{~m}$, was suspended vertically above the sand impact apparatus by a nylon thread attached to the distal end. The pressure loading on the impact end of the bar was recorded using a pair of foil strain gauges in a half Wheatstone bridge configuration. The gauges were mounted diametrically opposite each other and were oriented parallel to the bar axis, at a distance of approximately ten bar diameters from the impact end. Results were recorded using a digital storage oscilloscope at a sampling frequency of $500 \mathrm{kHz}$. The bar was calibrated by impacting the bar axially with a striker of identical diameter and material. The compressive pulse, as calculated using linear elastic wave theory, agreed well with the values obtained from the strain gauges. The calibration tests also indicated a rise time of approximately $13 \mu$ s for the dynamic strain measurements. Note that the Kolsky bar measurements have a duration of $0.43 \mathrm{~ms}$, after which time reflections from the distal end complicate interpretation of the results. In all cases, high speed photography confirmed that there was negligible motion of the Kolsky bar during the sand impact. For all results reported here, the Kolsky bar was aligned with the central-axis of the apparatus : spatial variations in the pressure loading were not investigated.

\subsection{Pressure and impulse measurements: dry sand}

The measured time variation of the pressure $p$ exerted on the end of the Kolsky bar by the sand impact is shown in Fig. 5a for dry sand with depth of burial $D=15 \mathrm{~mm}$ and stand-off distance $S=15 \mathrm{~mm}$ (the distance $S$ is measured between the impacted end of the Kolsky bar and the top surface of the sand). Two measurements are shown, to give an indication of repeatability. Two phases of pressure loading are apparent: an initial high pressure transient phase, followed by a longer duration period of approximately constant pressure. For both cases shown, the velocity of the sand on impact with the Kolsky bar is $v_{0} \approx 39 \mathrm{~ms}^{-1}$ (obtained from Fig. 3 for a stand-off $S=$ $15 \mathrm{~mm})$. Upon taking the density to be $\rho_{D}=1630 \mathrm{kgm}^{-3}$, the measured pressure during the low pressure phase of $2.5 \mathrm{MPa}$ is on the order of $\rho_{D} v_{0}^{2}$. This is consistent with steady lateral flow of the sand against the Kosky bar $\left(p=\rho_{D} v_{0}^{2}\right.$ is obtained by considering conservation of momentum for this sand flow). 
During the initial transient phase the peak pressure is of the order $8 \rho_{D} v_{0}{ }^{2}$. This transient phase has been analysed by Pingle et al. (2012), and can be attributed to a compaction front developing at the point of impact. The latter will occur if the density of the sand reduces during the bubble expansion phase, i.e. the density at impact $\rho_{i}<\rho_{D}$. The numerical analysis of an expanding spherical shell of dry sand by Deshpande et al. (2009) predicts such a density reduction, such that the shell increases in thickness due to particle collisions. Treating the sand as a rigid-ideally plastic-locking solid of negligible strength, which has linear viscosity $\eta$, Pingle et al. (2012) predict the duration of the transient to depend on $\rho_{i} / \rho_{D}$ as follows

$$
\tau=\frac{\left(1-\frac{\rho_{i}}{\rho_{D}}\right)^{2} \eta \pi}{4 v_{0}^{2} \rho_{i}} .
$$

The accumulated impulse per unit area $I$ imparted by the sand is calculated from the pressure as follows:

$$
I(t)=\int_{0}^{t} p\left(t^{\prime}\right) d t^{\prime}
$$

The result for dry sand is plotted in Fig. 5b. The rapid initial rise in imparted impulse corresponds to the early peak in pressure. The imparted impulse continues to rise at a lower rate during the pressure plateau phase. Although the pressure is low in this phase, the duration of loading is long, and so the total impulse imparted in this phase is significant.

\subsection{Pressure and impulse measurements: wet sand}

Results for wet sand are shown in Fig. 6. Again, the depth of burial and stand-off are $D=15 \mathrm{~mm}$ and $S=15 \mathrm{~mm}$, respectively, and two repeat measurements are shown. Pressure versus time is shown in Fig. 6a. As with the dry sand case, there is an initial peak in pressure which drops off to a lower pressure plateau of longer duration. The maximum pressure is higher in the wet sand case compared to the dry, and the duration of the initial high pressure transient is also shorter. The wet sand is of measured density $\rho_{W}=2020 \mathrm{kgm}^{-3}$, and impact velocity is $v_{0}=31 \mathrm{~ms}^{-1}$ (from Fig. 3). Consequently, the measured peak pressure is approximately $13 \rho_{W} v_{0}^{2}$ and the duration of the transient is approximately $0.1 \mathrm{D} / v_{0}$. Deshpande et al. (2009) also analysed the expansion of a spherical shell of wet shell, and they show that, unlike dry sand, its density remains almost constant during the motion. The higher density and higher elastic stiffness of the wet sand compared to those of the dry sand contribute to the differences in pressure transient. The accumulated impulse with time for the wet sand is shown in Fig. 6b. The impulse imparted in the early phase of loading is lower than 
in the dry case, due to the short duration of the high pressure phase. However, the total impulse imparted over longer times exceeds that of the dry sand.

\subsection{The effect of stand-off}

Empirical models for the impulse imparted by a buried explosive indicate sensitivity to the stand-off distance. In this section we use the Kolsky bar to study the effect of stand-off for the sand impact apparatus. Here, we define the stand-off $S$ to be the distance between the top surface of the soil and the end of the Kolsky bar, which is again located centrally with respect to the buried charge simulator. The sand depth $D$ equals $15 \mathrm{~mm}$ and the stand-off $S$ is varied in the range $15-60 \mathrm{~mm}$. Both dry and fully saturated wet sand are employed in these tests.

To allow comparison between the sand impact loading at each stand-off distance, we consider two metrics: the peak pressure over the measured time-history $p_{m}$ and the total imparted impulse per unit area $I_{t}$. As noted above, the duration of the recorded pressure-time response is limited in these experiments by the length of the Kolksy bar. In some experiments, the tail of the pressure time history is truncated by reflections from the distal end. To provide a consistent measure of the total impulse we define

$$
I_{t}=\int_{0}^{D / v_{0}} p d t
$$

where $v_{0}$ is the velocity of the sand front at impact with the Kolsky bar, as obtained from the high speed photography. Given that the pressure falls to a small fraction of the peak value by $t=D / v_{0}$ in all cases, it is reasonable to assume that $I_{t} \approx I(t=\infty)$.

The results are plotted in Fig. 7, for dry and fully saturated wet sand. Each point corresponds to an individual measurement; repeat measurements are shown for each condition to demonstrate the consistency of the results. For both wet and dry sand, the peak pressure increases with stand-off up to a distance $S / D \approx 2$. Further increasing the stand-off reduces the peak pressure. Wet sand exerts a higher peak pressure than dry over the full range of stand-off. The maximum in peak pressure can be explained by the competition between two effects. Firstly, the sand layer is accelerating up to a stand-off of approximately $S / D \approx 3$ (Fig. 3). Secondly, the density of the sand flow reduces as the stand-off increases, as it develops from a dense shell into a loose spray (Fig. 2).

For both wet and dry sand, the total imparted impulse reduces approximately linearly with stand-off, and is also higher for the wet sand across the full range. The total impulse is dictated by two effects: the mass per unit area of sand impacting the 
Kolsky bar scales as $S^{-2}$ for a spherical shell expansion, and the velocity of the sand is linear in $S$, recall Fig. 3.

\subsection{The effect of soil over-burden}

The soil over-burden (the depth of burial) influences the loading imparted by a buried explosive (Bergeron et al., 1998). To examine the effect of soil over-burden, we now fix the stand-off at $S=30 \mathrm{~mm}$ and vary the depth of the sand layer $D$ in the range $15-30 \mathrm{~mm}$. Again, both dry and fully saturated wet sand are considered. The results are given in Fig. 8. For both wet and dry sand, the peak pressure reduces approximately linearly with increasing $D$, and the wet sand exerts a higher peak pressure over the full range. This is consistent with the lower impact speeds for deeper sand (Fig. 3). The total imparted impulse is however insensitive to $D$ (Fig. 7b). This insensitivity, compared to the stand-off effect, is consistent with the empirical model of Tremblay (1998). In qualitative terms, the mass of sand impacting the Kolsky bar increases with $D$, this is balanced by the reduction in impact velocity.

\section{Preliminary assessment of fluid-structure interaction effects}

Fluid-structure interaction effects have been investigated recently in the development of light-weight materials to protect against an underwater blast (Fleck and Deshpande, 2004, McShane et al., 2007, Liang et al., 2007). Reducing the mass of the target is observed to reduce the imparted momentum. The experiments of Fourney et al. (2005) indicate that fluid-structure interaction effects may also be important during loading by sand impact. Using reduced scale landmine experiments (using $609 \times 10^{-6} \mathrm{~kg}$ TNT charges, corresponding to approximately 10 times the energy release of the experiments performed here), their results indicate that the momentum imparted to a vertical impulse pendulum is influenced by the pendulum mass. In this section we employ a vertical impulse pendulum of variable mass to assess fluidstructure interaction effect for sand impacts using the laboratory scale apparatus.

\subsection{The vertical impulse measurement apparatus}

The impulse pendulum assembly is shown in Fig. 9. The pendulum consists of a metallic circular cylinder of diameter $50 \mathrm{~mm}$ attached to a thin-walled nylon supporting tube of identical diameter. Various lengths of metallic cylinder are used, machined from either brass or aluminium, such that the mass is in the range 0.05$0.8 \mathrm{~kg}$. The nylon tube has a mass $0.1 \mathrm{~kg}$. The nylon tube and metallic cylinder slides freely within a transparent PMMA guiding tube which is held clamped above the sand impact apparatus. In all experiments the vertical impulse pendulum is positioned 
centrally with respect to the buried charge simulator. During the sand impact event, momentum is imparted to the pendulum and it proceeds to slide vertically within the PMMA tube. The pendulum diameter was selected so that the majority of the sand flow generated for a $15 \mathrm{~mm}$ thick sand layer will strike the pendulum - see Fig. 2. After impact by the sand, the vertical position of the pendulum is tracked over time using high speed photography. Equally spaced reference marks are machined into the nylon tube, to assist with the definition of instantaneous height. Neglecting sliding friction in the tube, the total imparted impulse per unit area is

$$
I_{t}=m_{p} \sqrt{2 g h_{\max }},
$$

where $m_{p}$ is the total pendulum mass per unit area (metal cylinder plus nylon tube) and $h_{\max }$ is the maximum height achieved by the pendulum during its motion. A frictional marker, fabricated from a split rubber o-ring, sits initially on top of the nylon tube. Sliding up with the pendulum, but not returning under gravity, it provides a secondary measure of $h_{\max }$. Excellent agreement was observed between the two methods of measurement.

\subsection{Experimental results}

Vertical impulse pendulum measurements were conducted using both dry and fully saturated wet sand with depth $D=15 \mathrm{~mm}$. In all cases the stand-off distance $S=$ $30 \mathrm{~mm}$, measured from the top surface of the sand to the lower face of the impulse pendulum. Examples of the time variation in the position of an impulse pendulum with total mass $0.66 \mathrm{~kg}$ are shown in Fig. 10, for both wet and dry sand impacts. Each point in Fig. 10 represents an individual frame from the high speed photography. The wet sand imparts a larger impulse to the pendulum than the dry: $I_{t}=0.55 \mathrm{kPas}$ (wet), $I_{t}=0.45 \mathrm{kPas}$ (dry). Fig. 11 shows the total imparted impulse as a function of the mass rato

$$
\bar{m}=\frac{m_{p}}{m_{s}},
$$

where $m_{p}$ is the pendulum mass per unit area. The mass of sand per unit area $m_{s}$ equals $D \rho_{D}$ for dry sand and equals $D \rho_{W}$ for wet sand. Selected repeat tests are included, and these show excellent repeatability. The improved repeatability compared to the Kolsky bar results is due to the larger cross-sectional area of the impulse pendulum, having the effect of averaging out small spatial variations in sand flow from test to test. The results show that the total imparted impulse is almost insensitive to pendulum mass for $\bar{m}>10$. For lighter targets, the imparted momentum reduces as the target mass reduces. 


\subsection{Discussion}

The effect of fluid content in the sand upon the imparted impulse is consistent with the findings from the Kolsky bar measurements. However, it should be noted that (even for large $\bar{m}$ ) the total imparted momentum obtained using the impulse pendulum is not directly comparable with the Kolsky bar measurements, due to the difference in the loaded area of the targets $(50 \mathrm{~mm}$ diameter, versus $12.6 \mathrm{~mm}$ diameter for the Kolksy bar). The impulse pendulum values are lower, consistent with a reduction in vertical impulse with increasing distance from the centre-line.

Deshpande et al. (2009) and Pingle et al. (2012) have previously analysed the onedimensional impact between a sand column and a target (either stationary or free to translate). They conclude that the interaction can be treated as an inelastic collision between two masses: the sand slug and the target. This motivates a first order model for the collision between the sand layer and the vertical impulse pendulum, as follows. Consider the incoming sand flow and pendulum as lumped masses $m_{s}$ and $m_{p}$, respectively, colliding inelastically. The average velocity of the sand before the collision is $v_{0}$, and the pendulum is initially at rest. After the collision is complete, the sand moves at average velocity $v_{s}$ and the pendulum at $v_{p}$. The overall coefficient of restitution for the collision reads

$$
e=\frac{v_{p}-v_{s}}{v_{0}} \text {. }
$$

(Note that this is distinct from the inter-particle coefficients of restitution within the sand.) Conservation of momentum leads to

$$
I_{t}=I_{0}(1+e) \frac{\bar{m}}{\bar{m}+1},
$$

where $I_{t}=m_{p} v_{p}$ is the imparted momentum and $I_{0}=m_{s} v_{0}$ is the initial momentum of the sand. The quantity $I_{0}(1+e)$ is the heavy plate limit of imparted momentum, and will be referred to subsequently as $I_{\infty}$. The upper limit $I_{\infty}=2 I_{0}$ is achieved for a fully elastic collision, and the lower limit $I_{\infty}=I_{0}$ is obtained in the case of a fully plastic impact.

The relationship (8) is included in Fig. 11, with the value of $I_{\infty}$ adjusted to fit the wet and dry experimental measurements at large $\bar{m}$. For the dry sand $I_{\infty(D)}=0.48 \mathrm{kPas}$ and for the wet $I_{\infty(W)}=0.59 \mathrm{kPas}$. This indicates a higher overall coefficient of restitution for the wet sand slug, assuming $I_{0}$ to be similar for wet and dry cases (although $I_{0}$ is difficult to evaluate precisely for the experimental sand flow). 
CASE? WHY NOT? NEED TO EXPLAIN THIS. The simple inelastic impact model seems to show reasonable agreement with the trends in the experimental data as $\bar{m}$ is reduced. Further study is required to fully evaluate the influence of key parameters such as soil depth, soil impact velocity and stand-off UPON WHAT GRAHAM??.

\section{Conclusions}

A new experimental technique has been developed to simulate the effects of landmine loading on structures in a laboratory setting. Suddenly discharged nitrogen gas is used to generate a dynamic flow of sand in a manner representative of a buried explosive. The method is flexible (sand depth, sand saturation and blast energy are easily controlled), has good repeatability and is amenable to instrumentation. It allows representative impact loading to be generated under laboratory conditions, without the need for explosives.

The apparatus has been used to study two sand impact phenomena: the time history of the pressure exerted on a Kolksy bar, and the total impulse imparted to a vertical impulse pendulum. The Kolsky bar measurements reveal two phases of pressure loading: a high pressure transient, followed by a low pressure phase of longer duration. The latter arises from the steady lateral flow of sand against the Kolsky bar. The peak pressure is higher for fully saturated wet sand compared to dry, and the high pressure transient phase is of shorter duration. This is consistent with the sand remaining clumped in a thinner, higher density shell during the 'bubble expansion' phase. The vertical impulse pendulum results confirm the higher total impulse imparted by wet sand impact compared to dry. In a simplified analysis, treating the sand slug and impulse pendulum as lumped masses and colliding inelastically, the larger impulse exerted by the wet sand indicates a more elastic collision, with a larger overall coefficient of restitution than that of the dry sand. 


\section{References}

Bagnold, R.A. (1954). Experiments on a gravity-free dispersion of large solid particles in a Newtonian fluid under shear. Proceedings of the Royal Society of London A 225, 49-63.

Bergeron, D, Walker, R., Coffey, C. (1998). Detonation of 100-gram anti-personnel mine surrogate charges in sand - A test case for computer code validation. Suffield Report No. 668, Defence Research Establishment Suffield, Ralston, Alberta, Canada.

Bolton, M.D. (1979). A guide to soil mechanics. Macmillan, London.

Braid, M.P. (2001). Experimental investigation and analysis of the effects of antipersonnel landmine blasts. Defence Research Establishment Suffield, Canada. Special Publication, DRES SSSP 2001-188.

Deshpande V.S., Heaver A., Fleck N.A. (2006). An underwater shock simulator. Proceedings of the Royal Society A 462, 1021-1041.

Deshpande, V.S., McMeeking, R.M., Wadley, H.N.G., Evans, A.G. (2009). Constitutive model for predicting dynamic interactions between soil ejecta and structural panels. Journal of the Mechanics and Physics of Solids 57, 1139-1164.

Dharan, C.K.H., Hauser, F.E. (1970). Testing techniques based on the split Hopkinson bar. Experimental Mechanics 10, 370-376.

Espinosa H.D., Lee S., Moldovan N. (2006). A novel fluid-structure interaction experiment to investigate deformation of structural elements subjected to impulsive loading. Experimental Mechanics 46, 805-824.

Fleck N.A., Deshpande V.S. (2004). The resistance of clamped sandwich beams to shock loading. Journal of Applied Mechanics 71, 386-401.

Fourney, W.L., Leiste, U., Bonenberger, R., Goodings, D. (2005). Explosive impulse on plates. Fragblast 9, 1-17.

Gujicic, M., Pandurangan, G., Cheeseman, B.A. (2006). The effect of degree of saturation of sand on detonation phenomena associated with shallow-buried and ground-laid mines. Journal of Shock and Vibration 13, 41-61.

Gujicic, M., Pandurangan, G., Qiao, R., Cheeseman, B.A., Roy, W.N., Skaggs, R.R., Gupta, R. (2008). Parametrization of the porous-material model for snad with different levels of water saturation. Soil Dynamics and Earthquake Engineering 28, 20-35.

Hlady, S.L. (2004). Effect of soil parameters on land mine blast. $18^{\text {th }}$ Military Aspects of Blast and Shock (MABS) Conference, Bad Reichenhall, Germany. 
Kambouchev, N., Noels, L., Radovitzky, R. (2006). Nonlinear compressibility effects in fluid-structure interaction and their implications on the air-blast loading of structures. Journal of Applied Physics 100, 063519.

Lester, C. (1996). Protection of light skinned vehicles against landmines - a review. Defence Science and Technology Organisation Technical Report DSTO-TR-0310.

Liang Y., Spuskanyuk A.V., Flores S.E., Hayhurst D.R., Hutchinson J.W., McMeeking R.M., Evans A.G. (2007). The response of metallic sandwich panels to water blast. Journal of Applied Mechanics 74, 81-99.

McShane G.J., Deshpande V.S., Fleck N.A. (2007). The underwater blast resistance of metallic sandwich beams with prismatic lattice cores. Journal of Applied Mechanics 74, 352-364.

Neuberger, A., Peles, S., Rittel, D. (2007a). Scaling the response of circular plates subjected to large and close-range spherical explosions. Part I: Air blast loading. International Journal of Impact Engineering 34, 859-873.

Neuberger, A., Peles, S., Rittel, D. (2007b). Scaling the response of circular plates subjected to large and close-range spherical explosions. Part II: Buried charges. International Journal of Impact Engineering 34, 874-882.

Pingle, S.M., Fleck, N.A., Wadley, H.N.G., Deshpande, V.S. (2012). Discrete element calculations of the impact of a sand column against rigid structures. International Journal of Impact Engineering 45, 74-89.

Radford, D.D., Deshpande, V.S., Fleck, N.A. (2005). The use of metal foam projectiles to simulate shock loading on a structure. International Journal of Impact Engineering 31, 1152-1171.

Tabor, D. (1969). Gases, liquids and solids and other states of matter. Cambridge University Press, Cambridge.

Taylor, L.C., Skaggs, R.R, Gault, W. (2005). Vertical impulse measurements of mines buried in saturated sand. Fragblast 9, 19-28.

Tremblay, J.E. (1998). Impulse on blast deflectors from a landmine explosion. Defence Research Establishment Valcartier, Quebec, Technical Report DREV-TM9814.

Wadley H., Dharmasena K., Chen Y., Dudt P., Knight D., Charette R., Kiddy K (2008). Compressive response of multilayered pyramidal lattices during underwater shock loading. International Journal of Impact Engineering 35, 1102-1114.

Westine, P.S., Morris, B.L., Cox, P.A., Polch, E. (1985). Development of computer program for floor plate response from land mine explosions. Contract Report No. 1345 for US Army TACOM Research and Development Centre. 


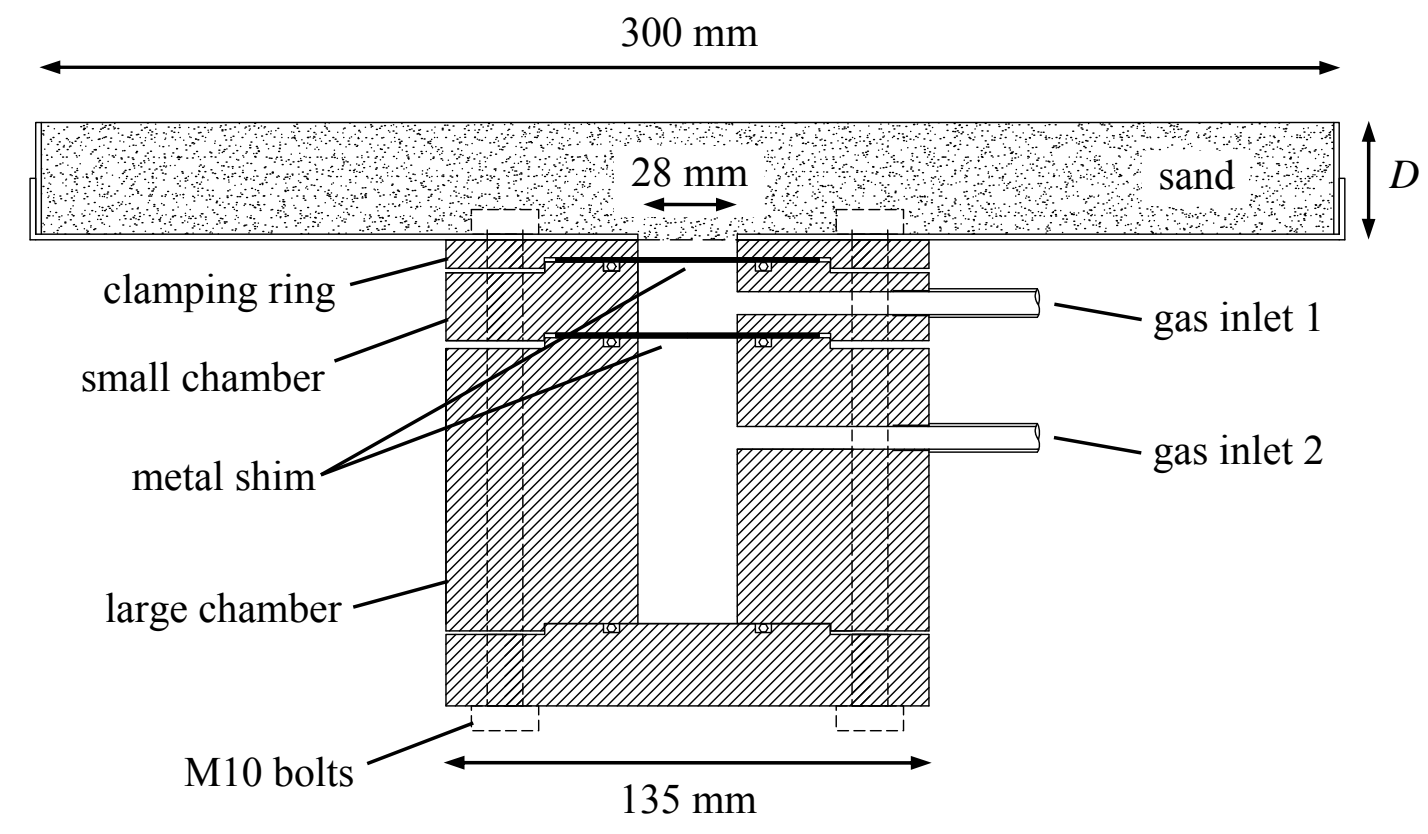

Figure 1: Cross-section through the apparatus. The pressure chambers, which are circular in plan view, have internal diameter $28 \mathrm{~mm}$ and heights $80 \mathrm{~mm}$ (large chamber) and $20 \mathrm{~mm}$ (small chamber). Rubber o-rings seal the joints between the stages of the pressure vessel. Metal shims are located between the large chamber and the small chamber, and between the small chamber and the clamping ring. A disc of aluminium foil (shown as a dashed line) seals the hole in the centre of the sand tray. 
(a)

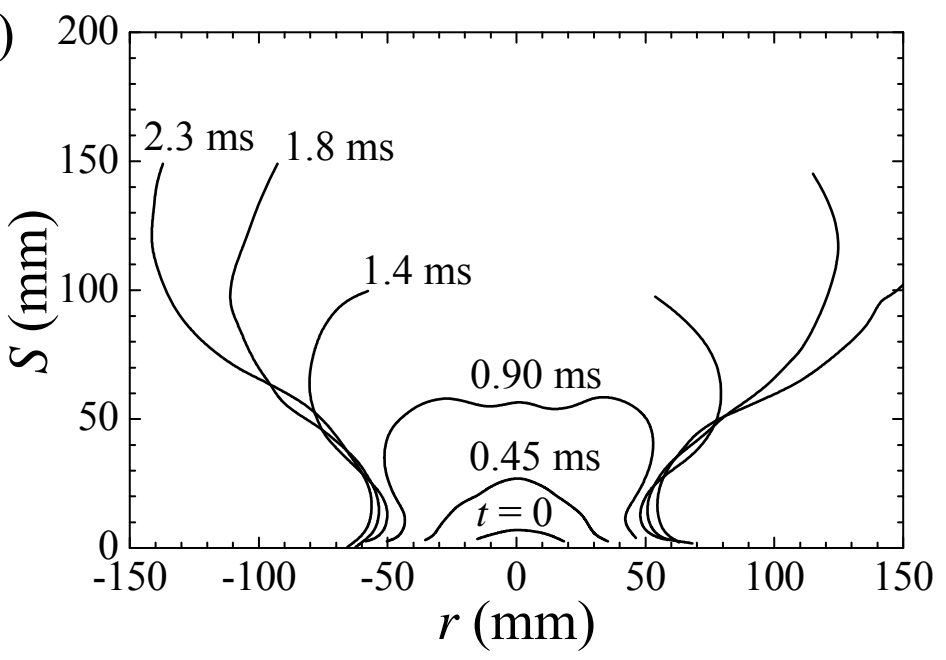

(b)

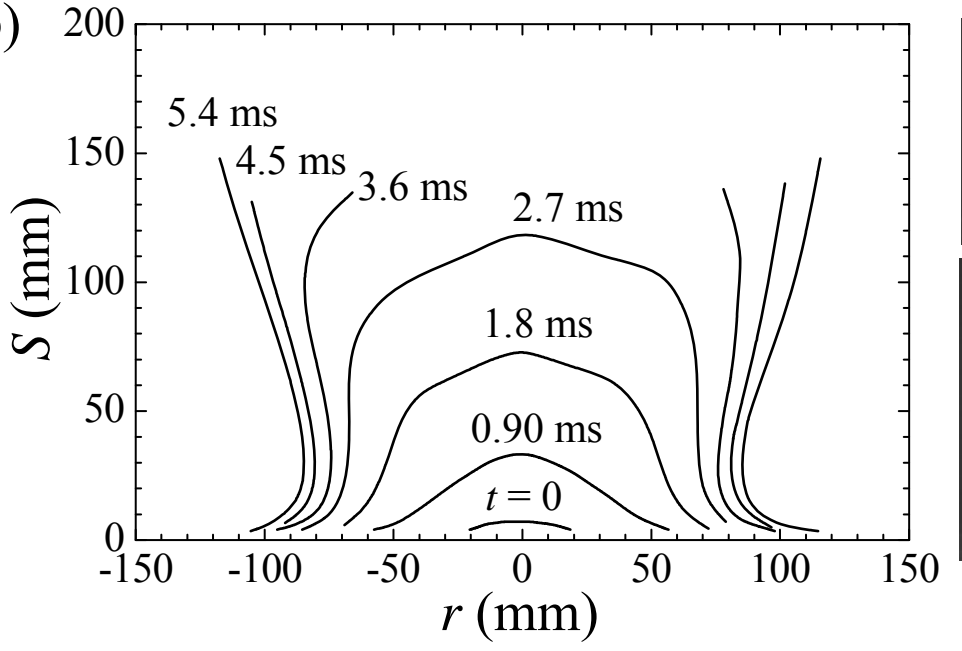

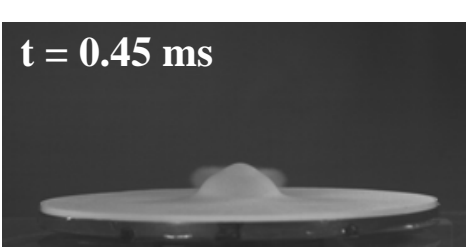
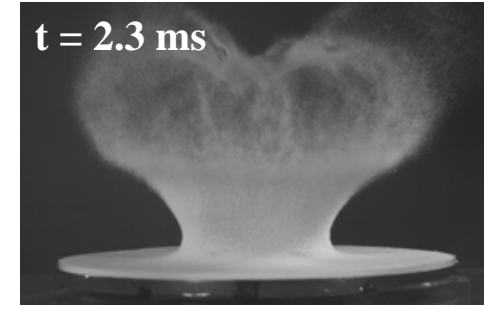

\section{$\mathrm{t}=0.90 \mathrm{~ms}$}
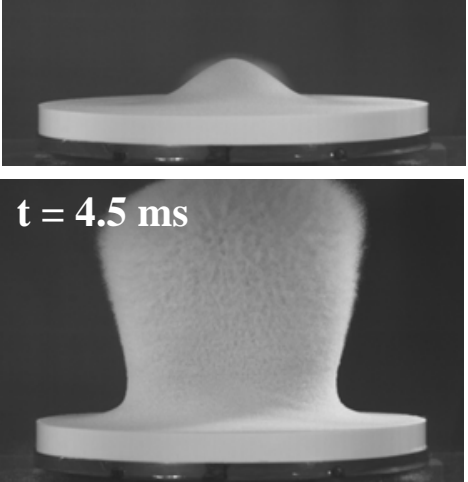

Figure 2: Measurements of the sand motion, from analysis of high speed photography. All results are for dry sand. Contours show the location of the sand front at various times for (a) $D=15 \mathrm{~mm}$ and (b) $D=30 \mathrm{~mm}$. $S$ is the height above the sand surface (the stand-off) and $r$ the radial distance from the centre of the sand tray. Selected frames from the high speed photography are shown. The times indicated are relative to the first frame in which motion of the sand surface was detected. 


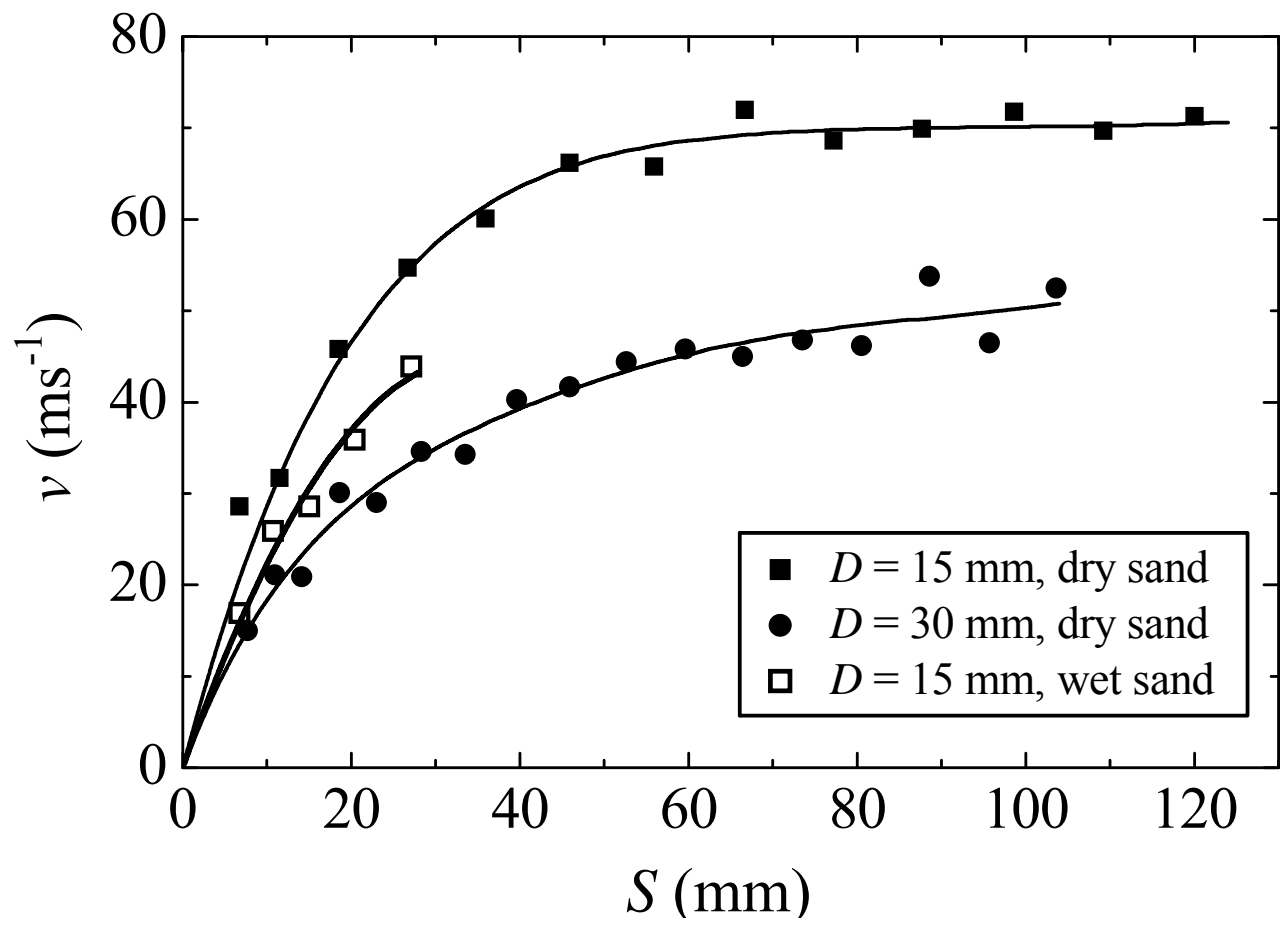

Figure 3: Variation with standoff $(S)$ of the velocity $(v)$ of the top surface of the sand at the centre-line of the apparatus. Points indicate individual measurements extracted from consecutive frames of the high speed photography. The solid lines are a fit through these points. 
(a)

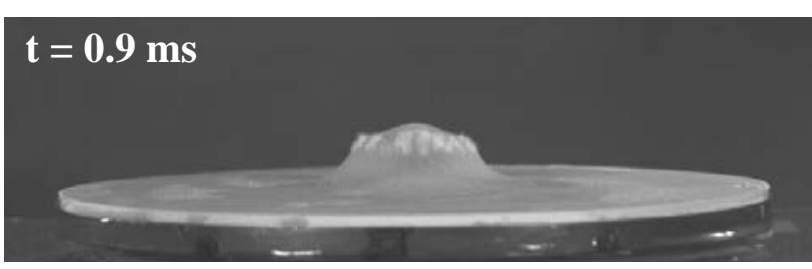

(b)

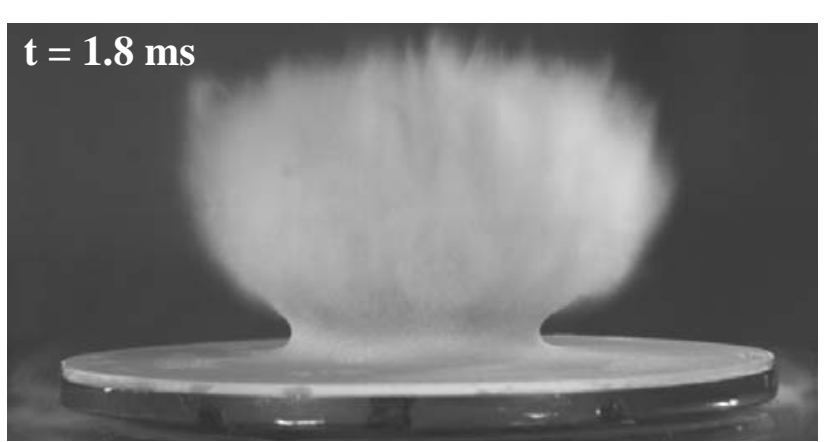

Figure 4: Selected frames from the high speed photography showing the flow of wet sand with depth of burial $D=15 \mathrm{~mm}$. The times indicated are relative to the first frame in which motion of the sand surface was detected. 
(a)

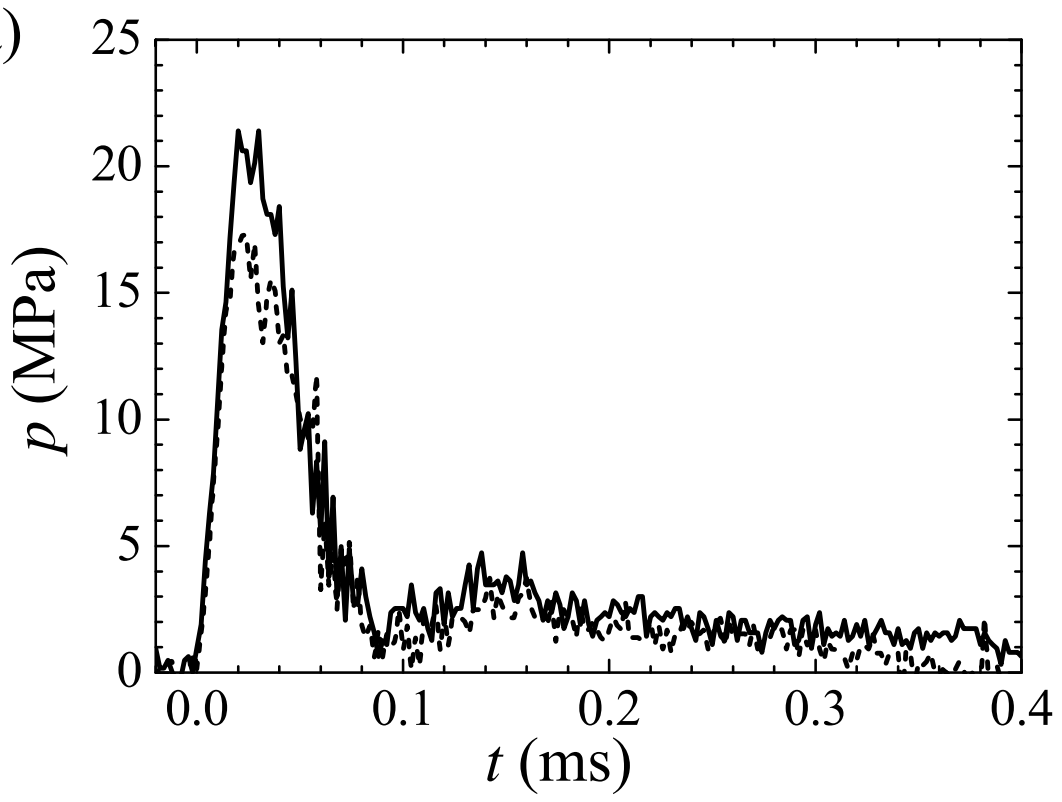

(b)

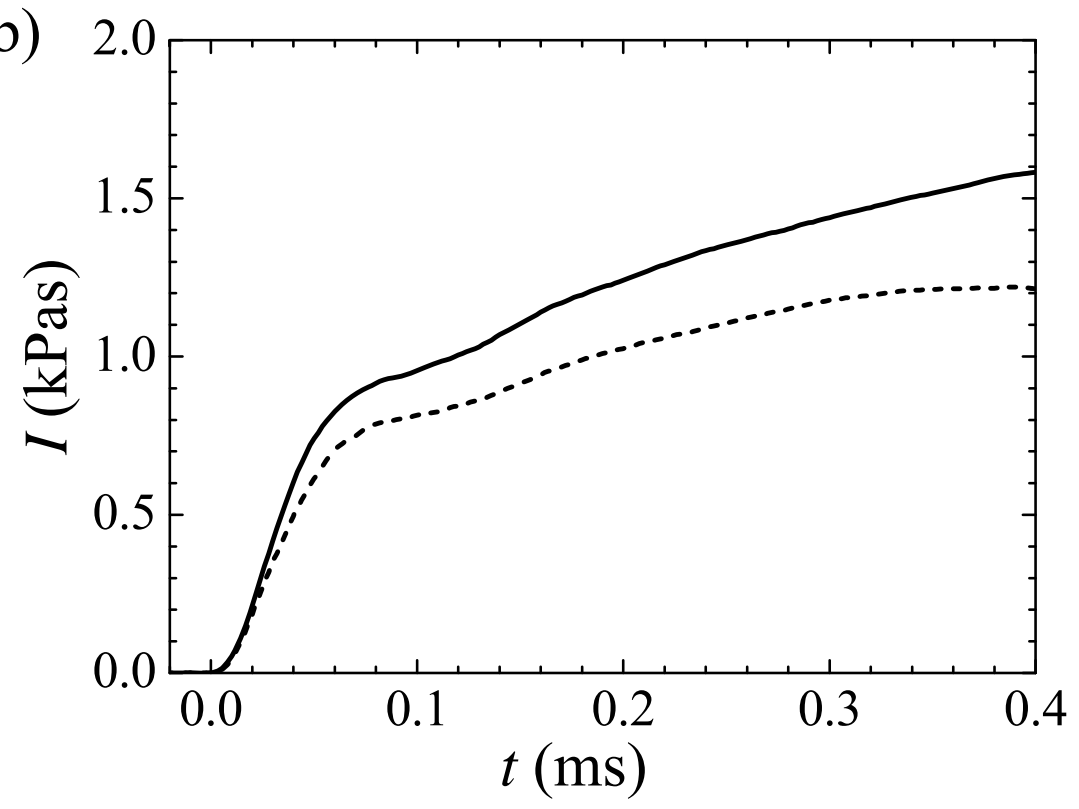

Figure 5: Measurements of (a) pressure $p$ and (b) transmitted impulse per unit area $I$ obtained using a Kolsky bar aligned with the centre-line of the apparatus. Results are for dry sand, with stand-off $S=15 \mathrm{~mm}$ and depth of burial $D=15 \mathrm{~mm}$. Two separate measurements are shown (indicated by solid and dashed lines). 
(a)

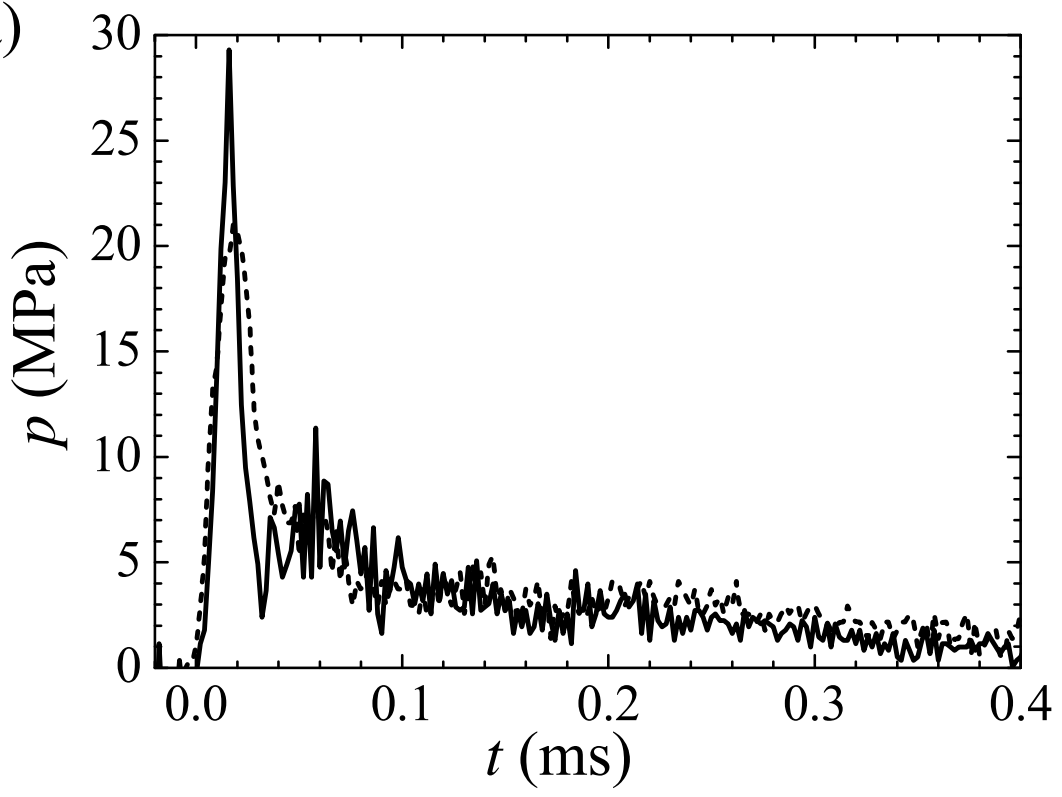

(b)

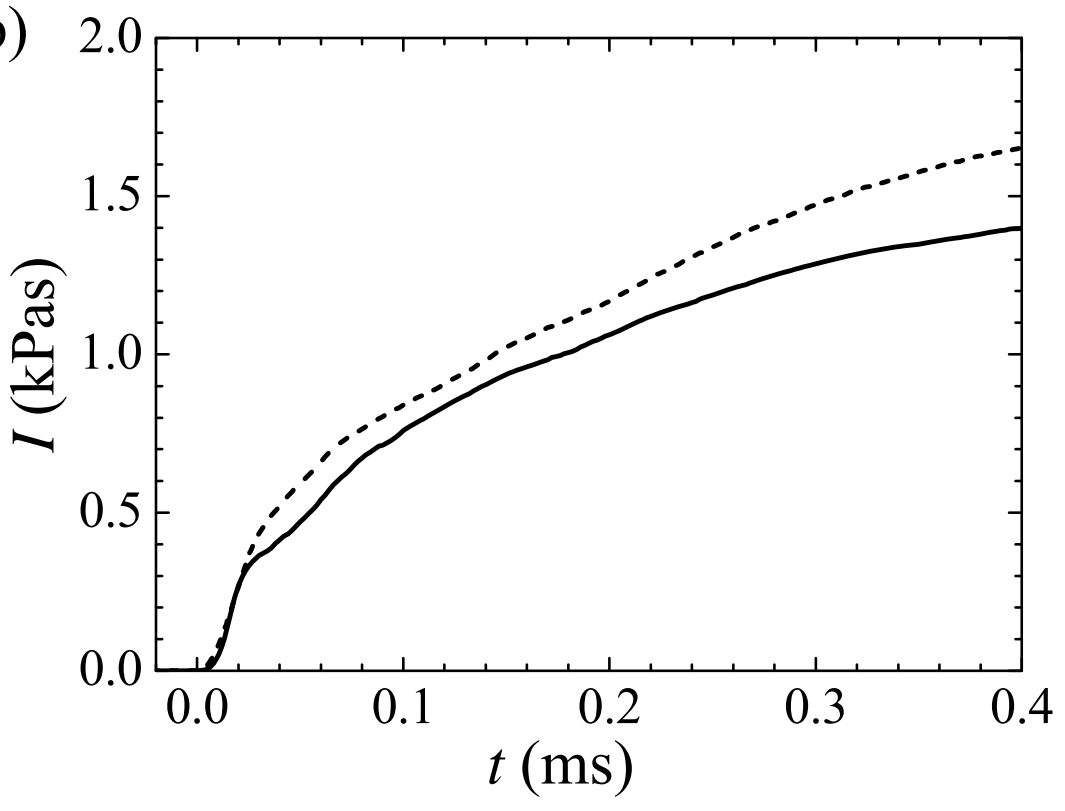

Figure 6: Measurements of (a) pressure $p$ and (b) transmitted impulse per unit area $I$ obtained using a Kolsky bar aligned with the centre-line of the apparatus. Results are for wet sand, with stand-off $S=15 \mathrm{~mm}$ and depth of burial $D=15 \mathrm{~mm}$. Two separate measurements are shown (indicated by solid and dashed lines). 
(a)

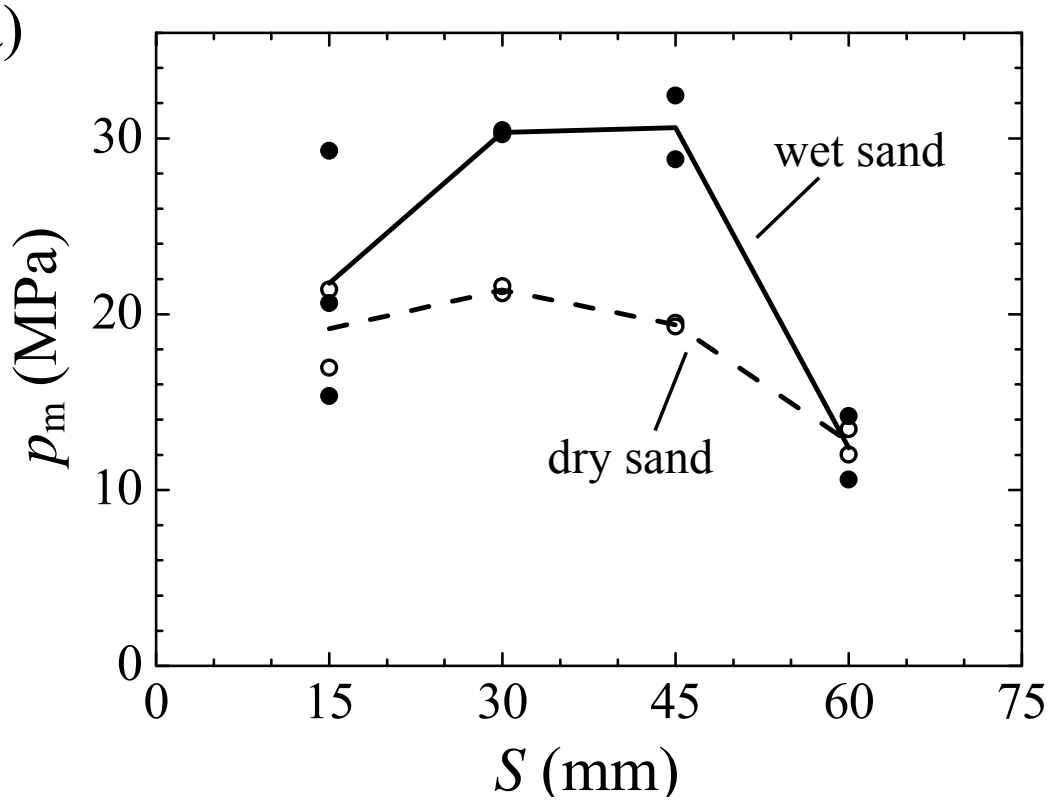

(b)

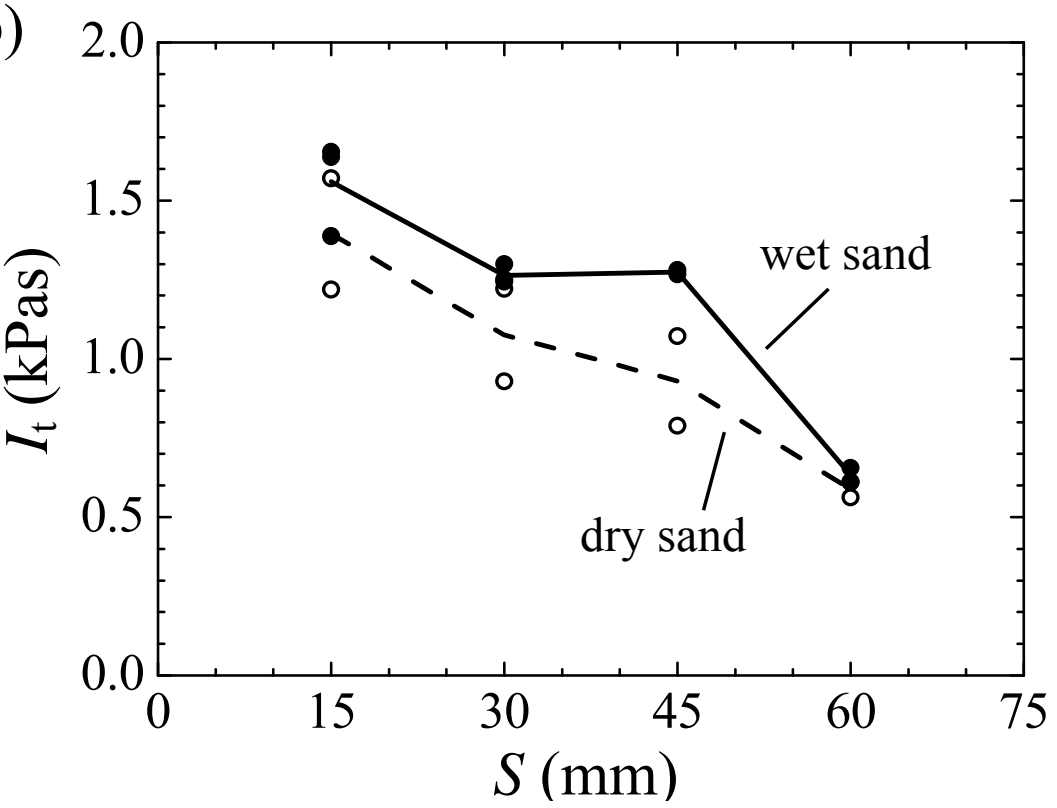

Figure 7: Variation with stand-off $S$ of (a) the peak pressure $p_{\mathrm{m}}$ and (b) the total transmitted impulse per unit area $I_{\mathrm{t}}$. The depth of burial $D=15 \mathrm{~mm}$. Points indicate individual measurements, the lines passing through the average values. 
(a)

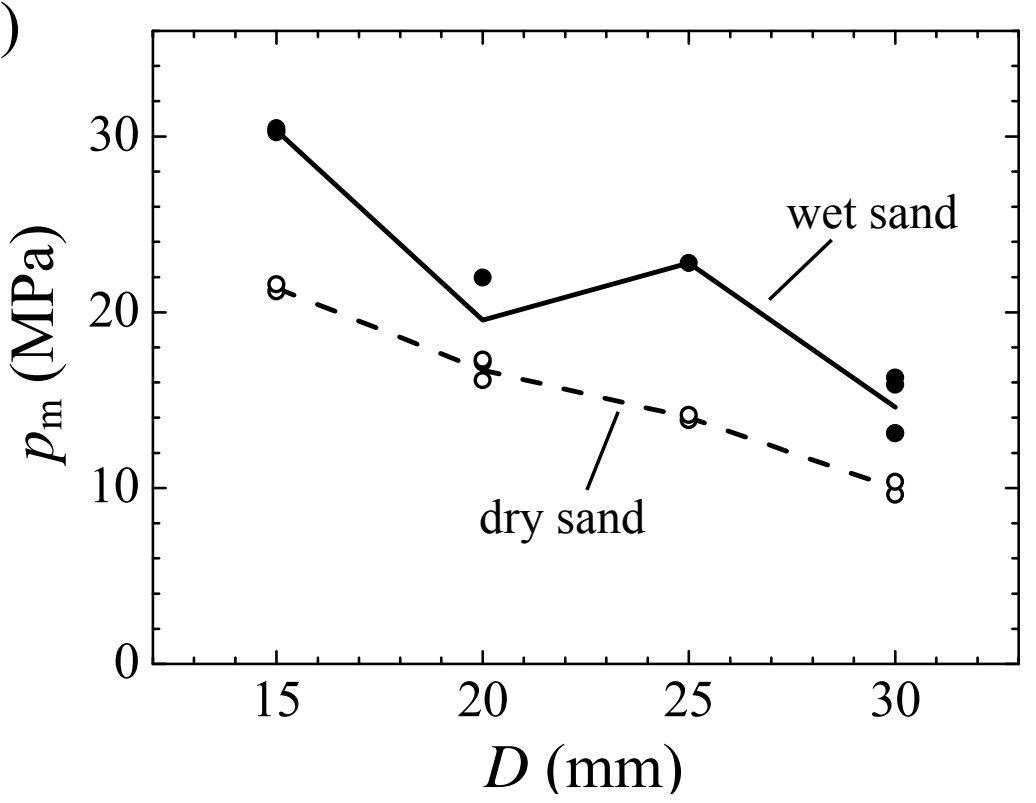

(b)

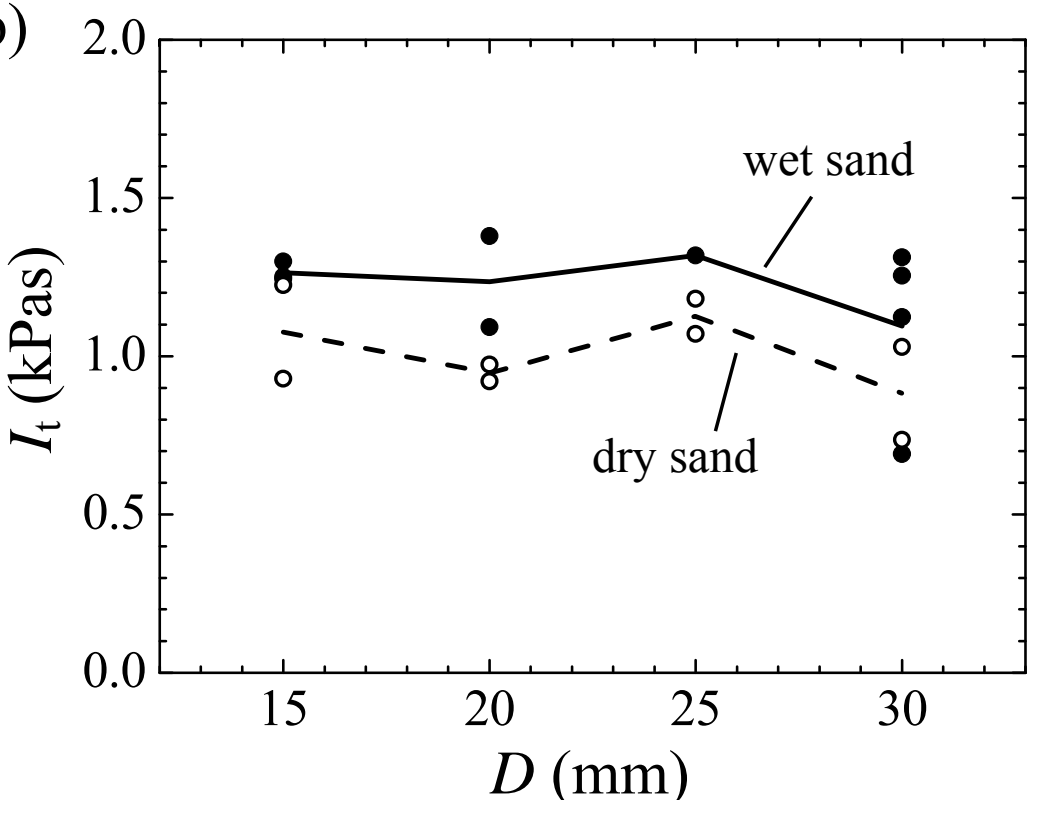

Figure 8: Variation with depth of burial $D$ of (a) the peak pressure $p_{\mathrm{m}}$ and (b) the total transmitted impulse per unit area $I_{\mathrm{t}}$. The stand-off $S=30 \mathrm{~mm}$. Points indicate individual measurements, the lines passing through the average values. 


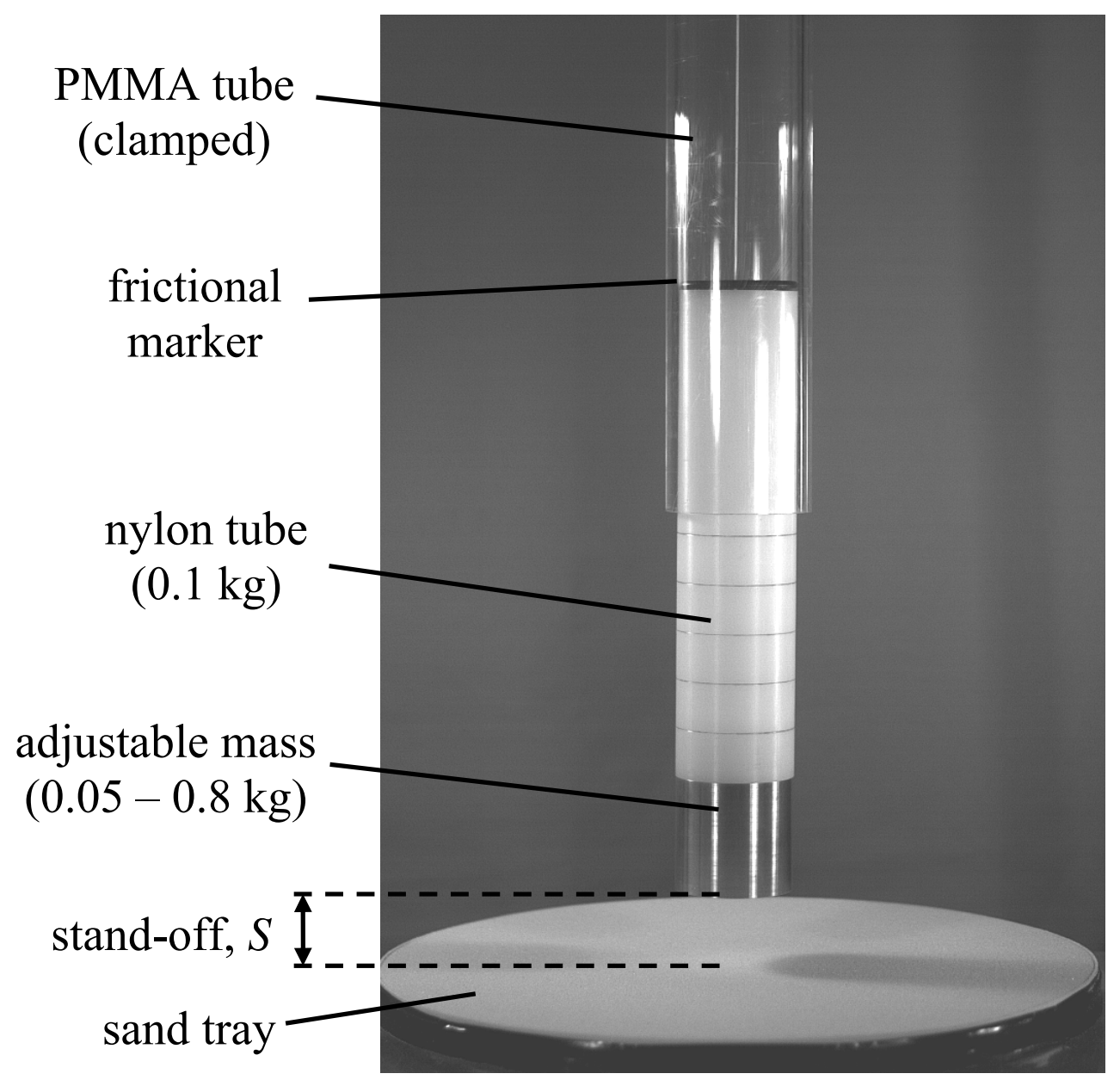

Figure 9: Photograph of the impulse pendulum apparatus. The pendulum consists of an adjustable mass of diameter $50 \mathrm{~mm}$, supported by a nylon tube, which is free to slide within a transparent PMMA tube. The position of the pendulum is monitored using high speed photography. A frictional marker confirms the maximum height obtained by the pendulum. 


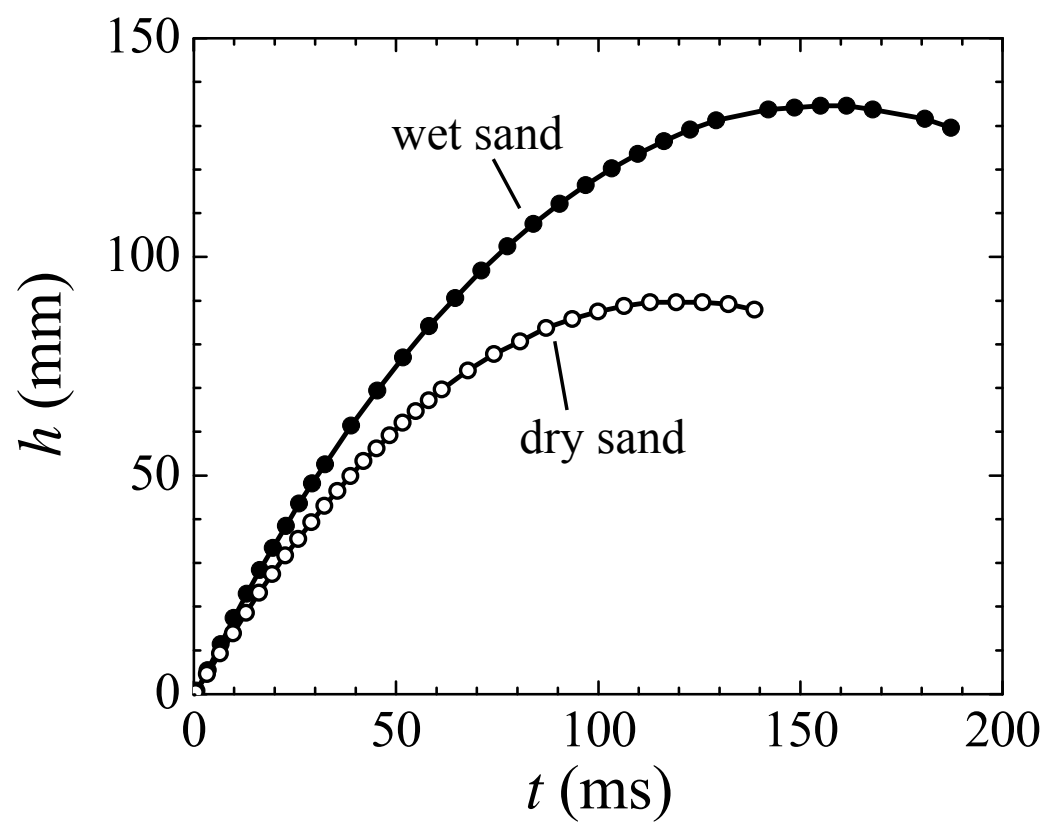

Figure 10: Vertical displacement of the impulse pendulum versus time, as measured from the high speed photography. The points denote individual measurements. Depth of burial $D=15 \mathrm{~mm}$, stand-off $S=30 \mathrm{~mm}$. The total mass of the impulse pendulum is $0.66 \mathrm{~kg}$.

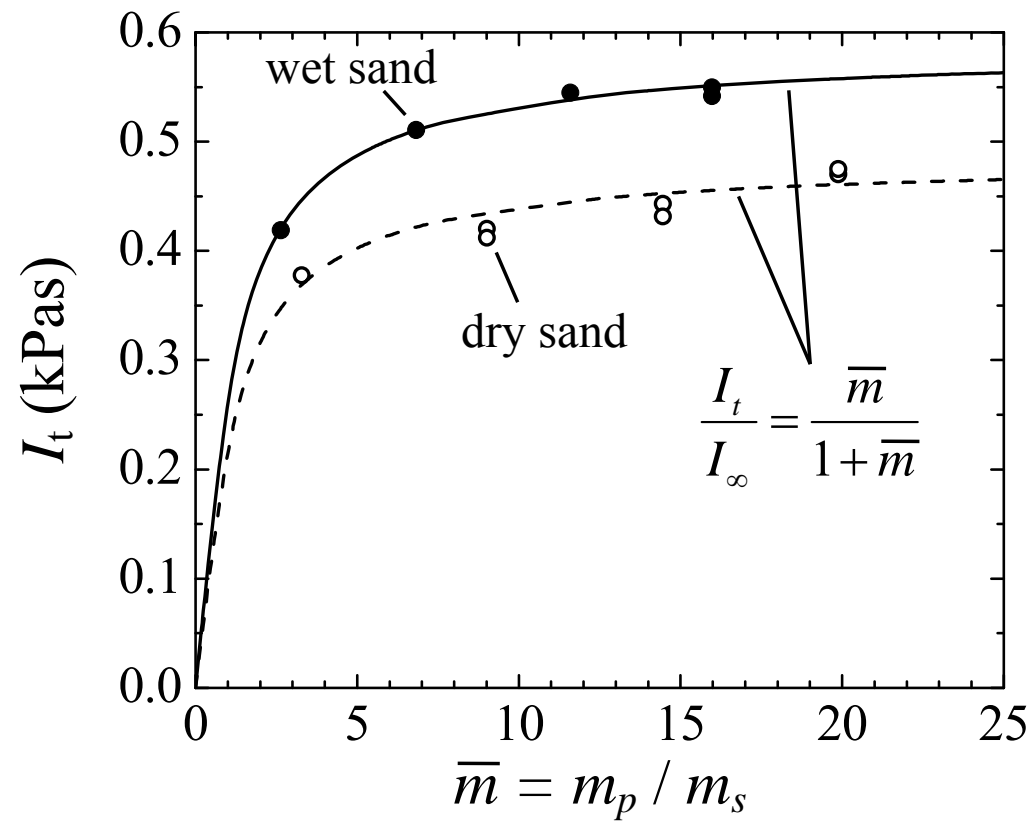

Figure 11: Variation in total transmitted impulse with the mass of the impulse pendulum. $\quad m_{p}$ and $m_{s}$ are the mass per unit area of the pendulum and sand, respectively. Points indicate individual experimental measurements. Depth of burial $D=15 \mathrm{~mm}$, stand-off $S=30 \mathrm{~mm}$. 


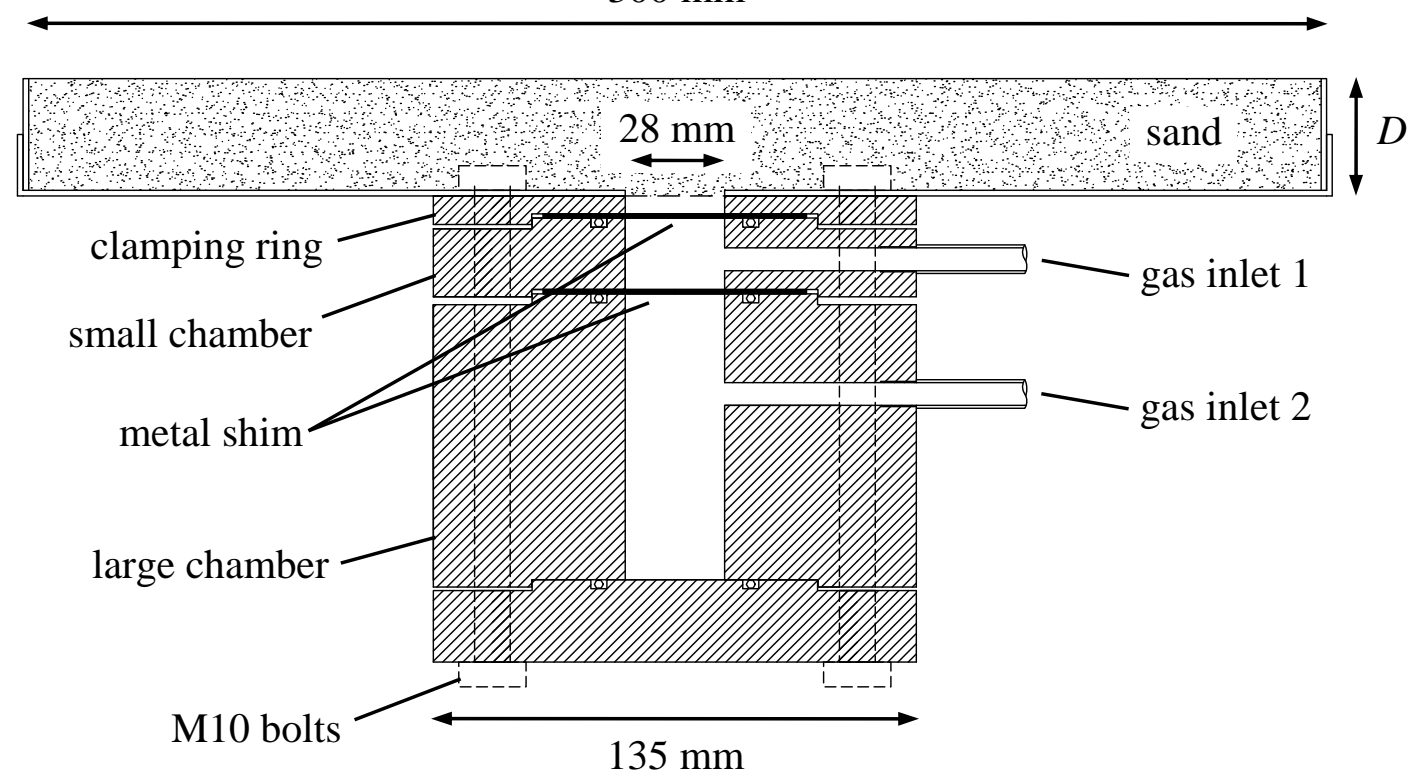

Figure 1: Cross-section through the apparatus. The pressure chambers, which are circular in plan view, have internal diameter $28 \mathrm{~mm}$ and heights $80 \mathrm{~mm}$ (large chamber) and $20 \mathrm{~mm}$ (small chamber). Rubber o-rings seal the joints between the stages of the pressure vessel. Metal shims are located between the large chamber and the small chamber, and between the small chamber and the clamping ring. A disc of aluminium foil (shown as a dashed line) seals the hole in the centre of the sand tray. 
(a)

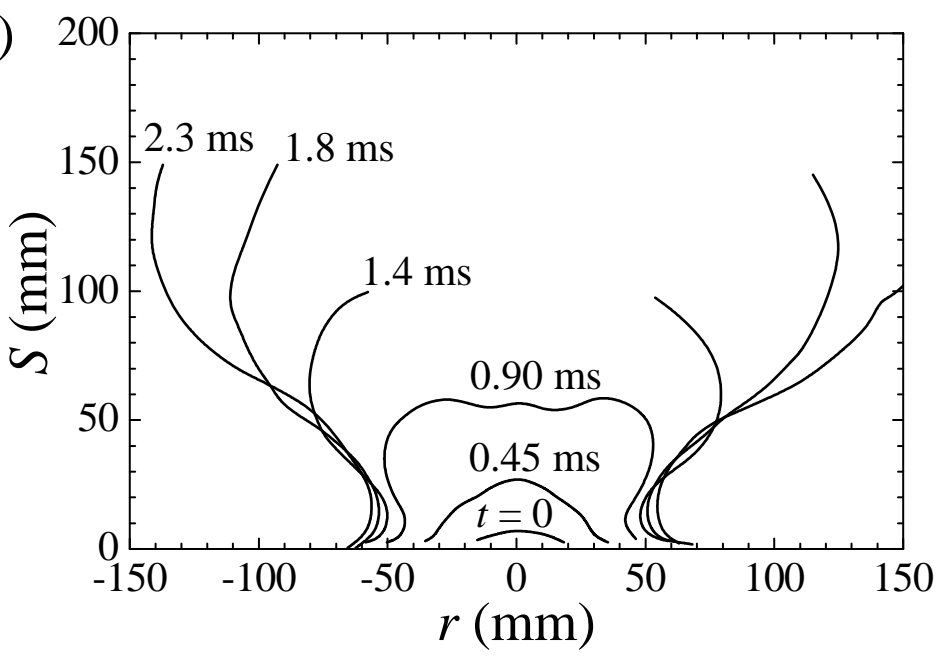

(b)

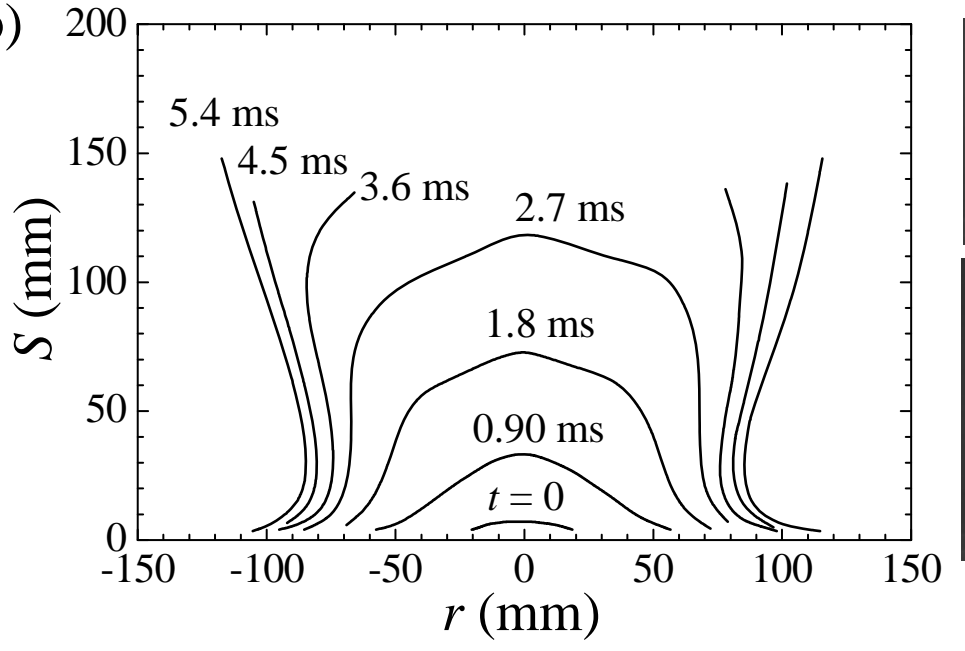

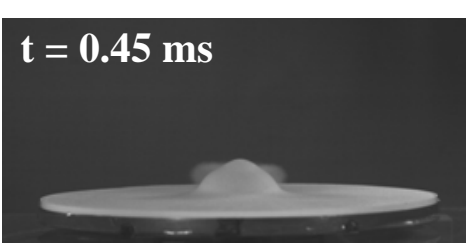
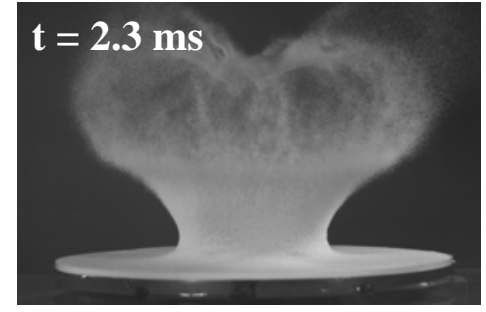

\section{$\mathrm{t}=\mathbf{0 . 9 0 \mathrm { ms }}$}
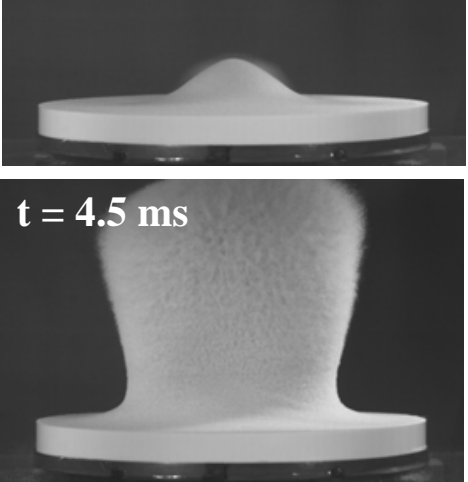

Figure 2: Measurements of the sand motion, from analysis of high speed photography. All results are for dry sand. Contours show the location of the sand front at various times for (a) $D=15 \mathrm{~mm}$ and (b) $D=30 \mathrm{~mm}$. $S$ is the height above the sand surface (the stand-off) and $r$ the radial distance from the centre of the sand tray. Selected frames from the high speed photography are shown. The times indicated are relative to the first frame in which motion of the sand surface was detected. 


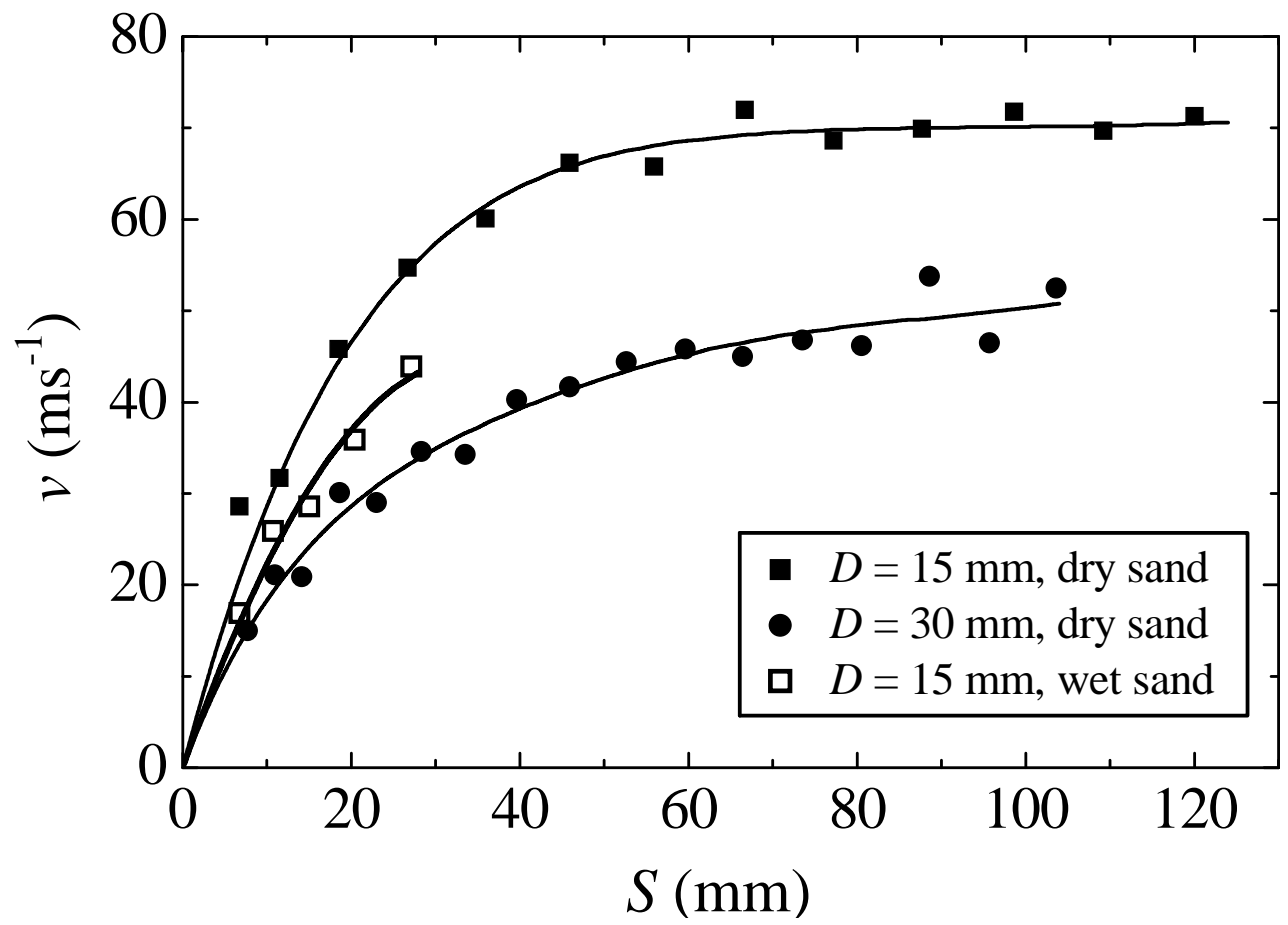

Figure 3: Variation with standoff $(S)$ of the velocity $(v)$ of the top surface of the sand at the centre-line of the apparatus. Points indicate individual measurements extracted from consecutive frames of the high speed photography. The solid lines are a fit through these points. 


\section{(a)}

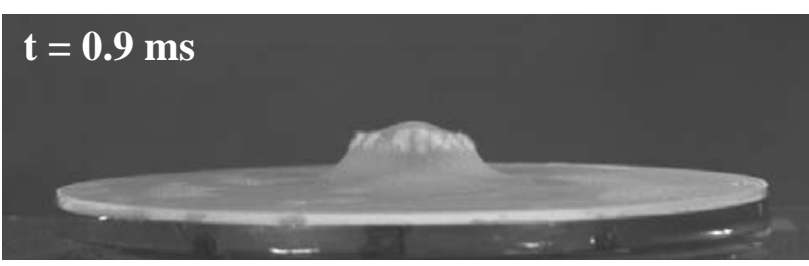

(b)

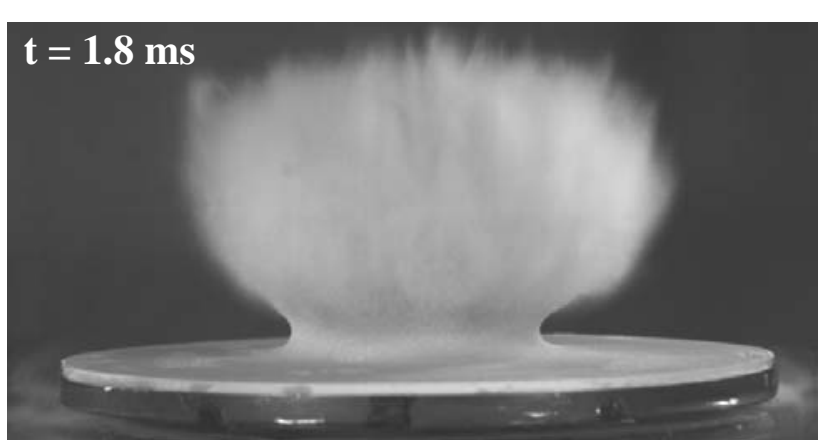

Figure 4: Selected frames from the high speed photography showing the flow of wet sand with depth of burial $D=15 \mathrm{~mm}$. The times indicated are relative to the first frame in which motion of the sand surface was detected. 

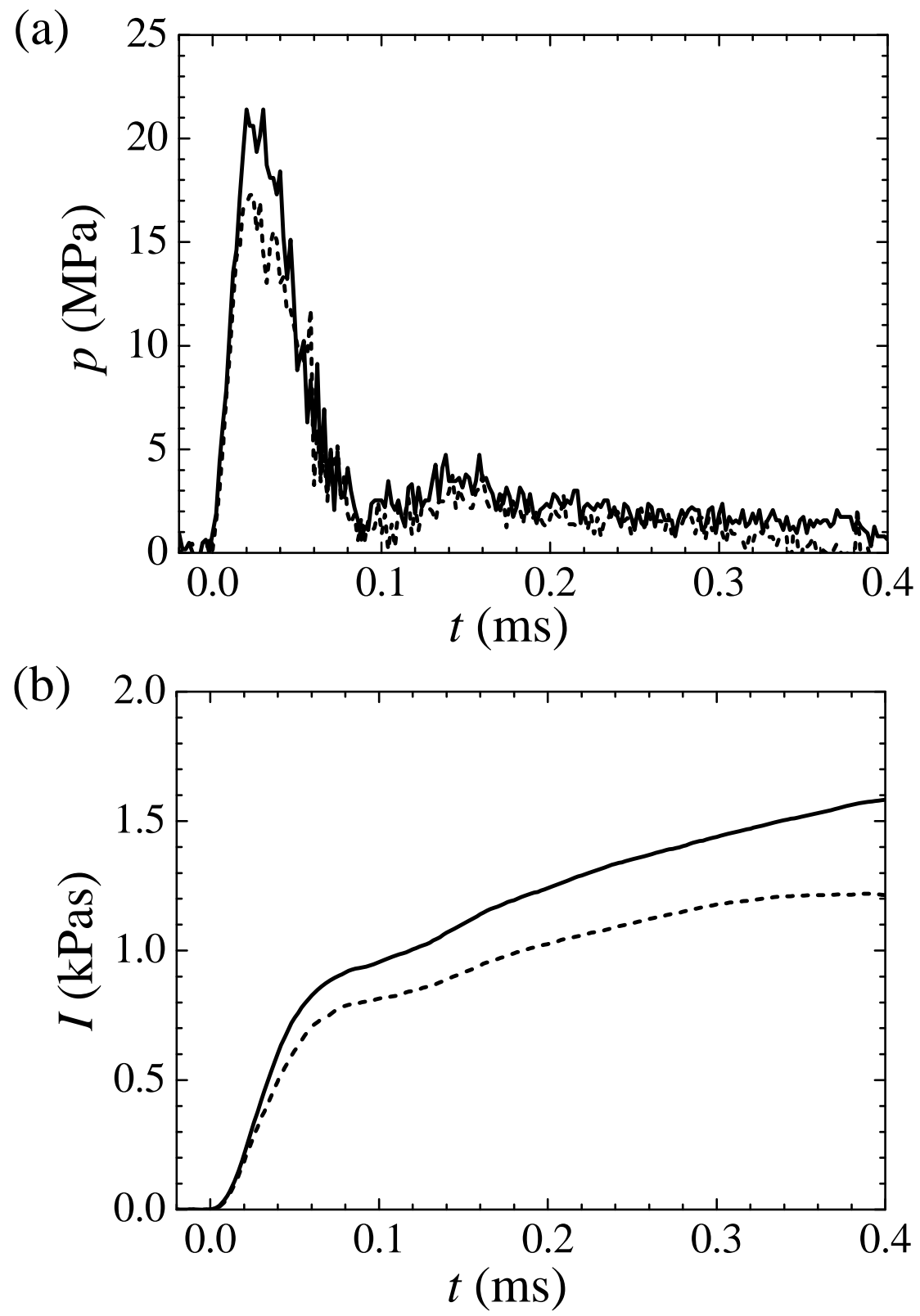

Figure 5: Measurements of (a) pressure $p$ and (b) transmitted impulse per unit area $I$ obtained using a Kolsky bar aligned with the centre-line of the apparatus. Results are for dry sand, with stand-off $S=15 \mathrm{~mm}$ and depth of burial $D=15 \mathrm{~mm}$. Two separate measurements are shown (indicated by solid and dashed lines). 
(a)
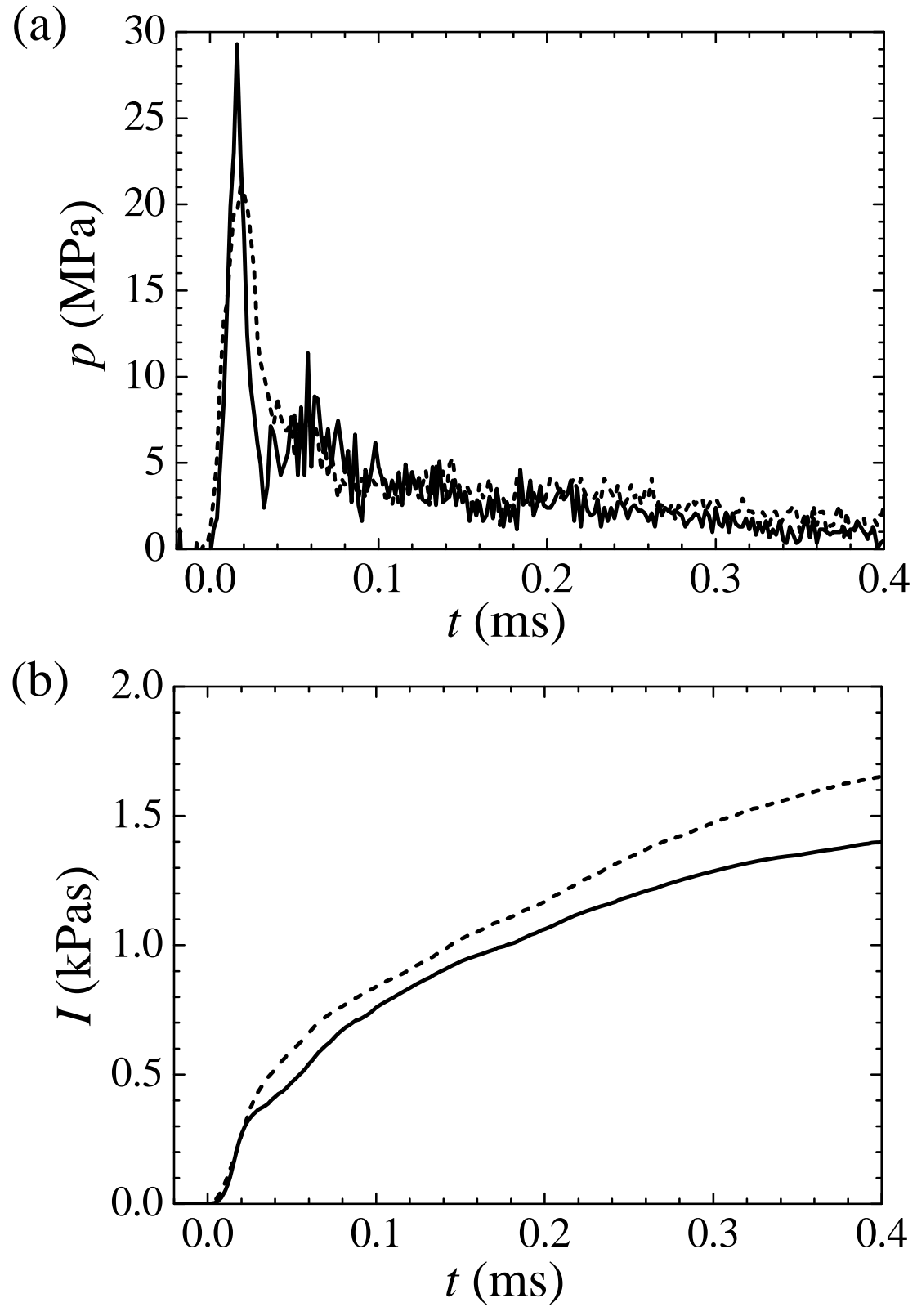

Figure 6: Measurements of (a) pressure $p$ and (b) transmitted impulse per unit area $I$ obtained using a Kolsky bar aligned with the centre-line of the apparatus. Results are for wet sand, with stand-off $S=15 \mathrm{~mm}$ and depth of burial $D=15 \mathrm{~mm}$. Two separate measurements are shown (indicated by solid and dashed lines). 
(a)

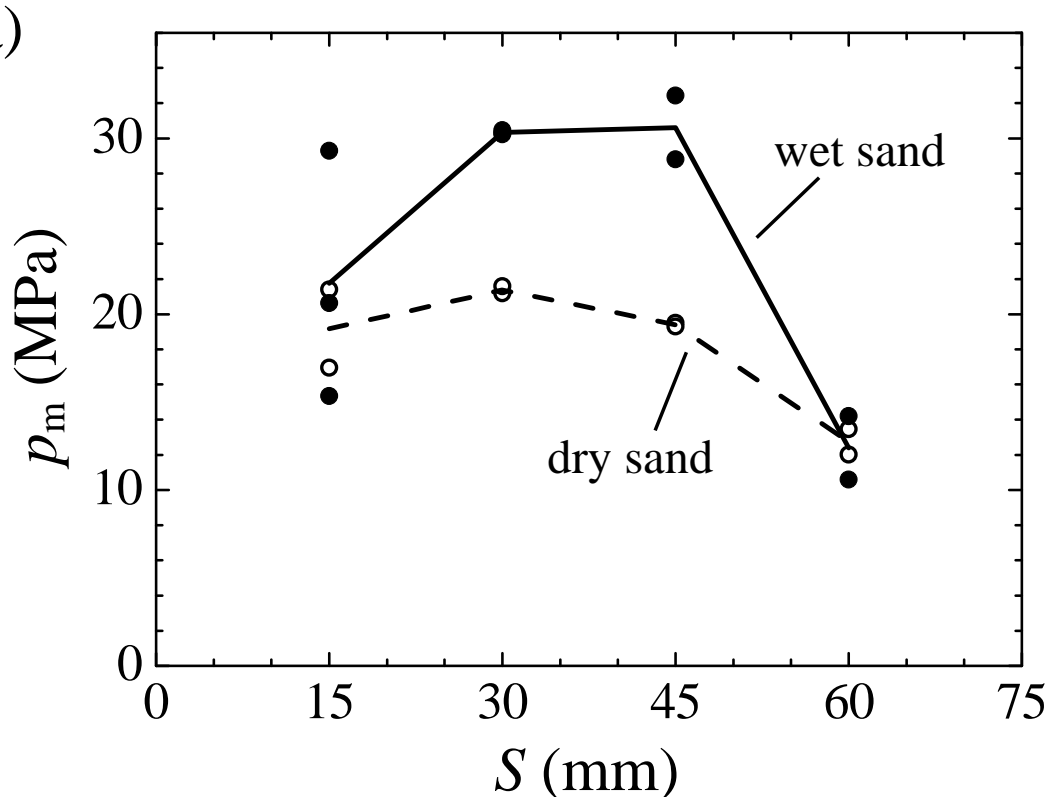

(b)

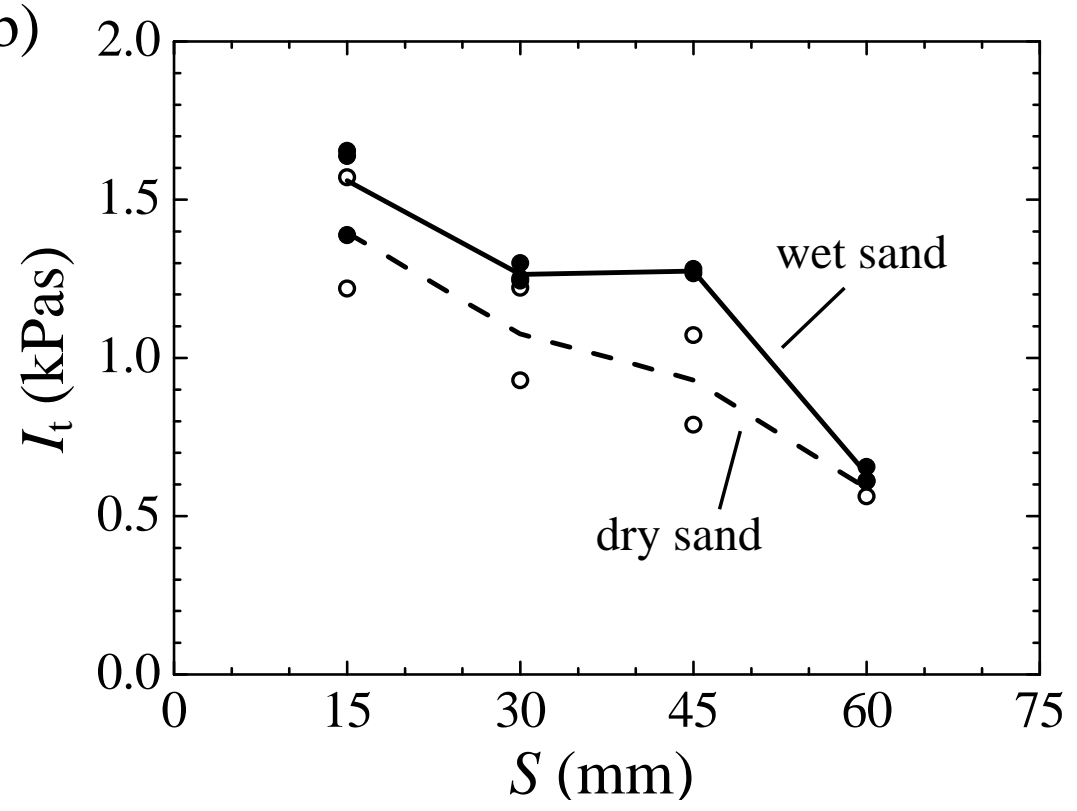

Figure 7: Variation with stand-off $S$ of (a) the peak pressure $p_{\mathrm{m}}$ and (b) the total transmitted impulse per unit area $I_{\mathrm{t}}$. The depth of burial $D=15 \mathrm{~mm}$. Points indicate individual measurements, the lines passing through the average values. 
(a)

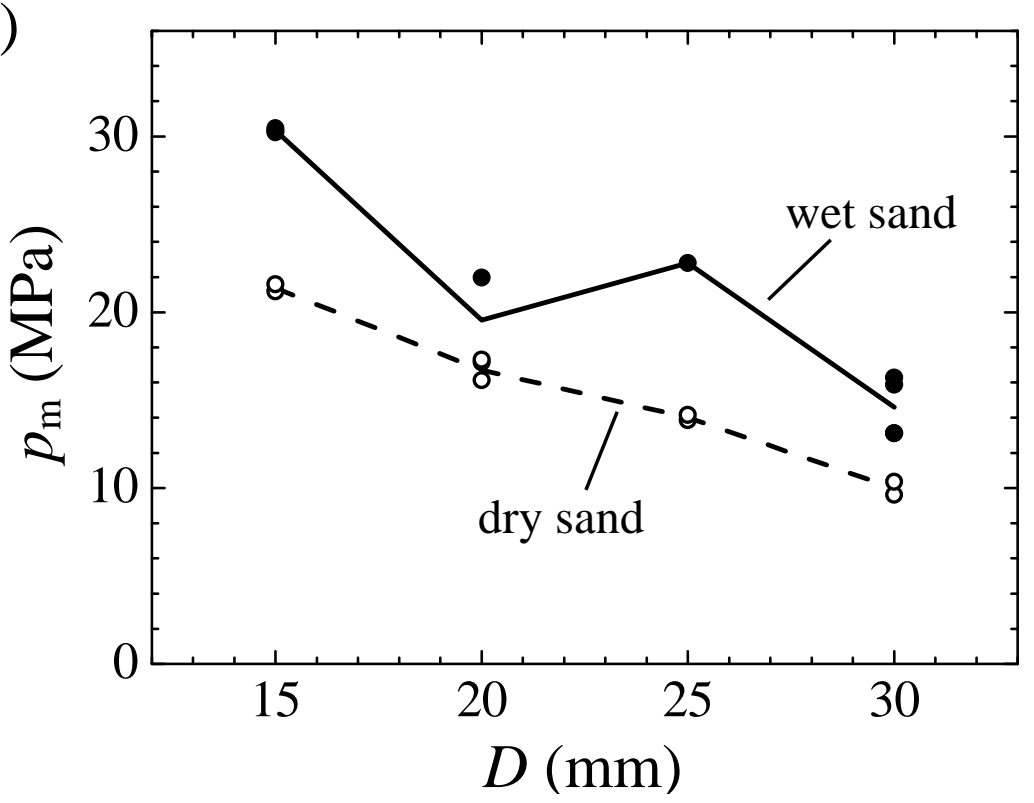

(b)

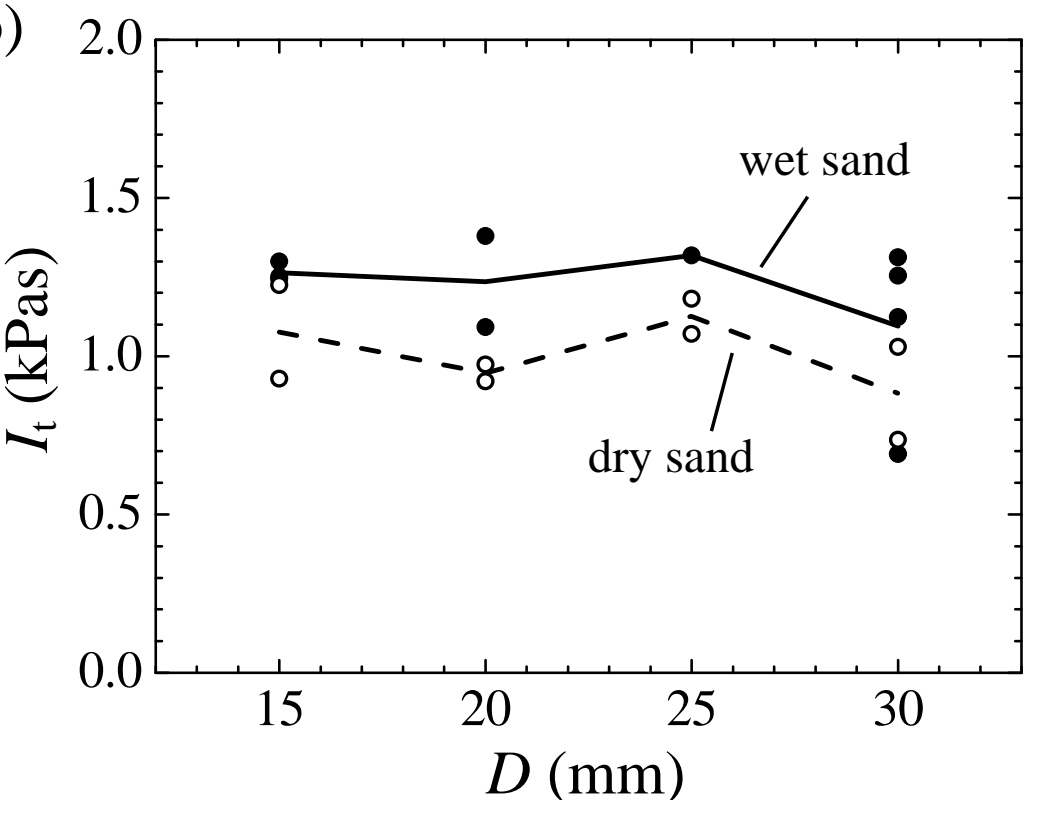

Figure 8: Variation with depth of burial $D$ of (a) the peak pressure $p_{\mathrm{m}}$ and (b) the total transmitted impulse per unit area $I_{\mathrm{t}}$. The stand-off $S=30 \mathrm{~mm}$. Points indicate individual measurements, the lines passing through the average values. 


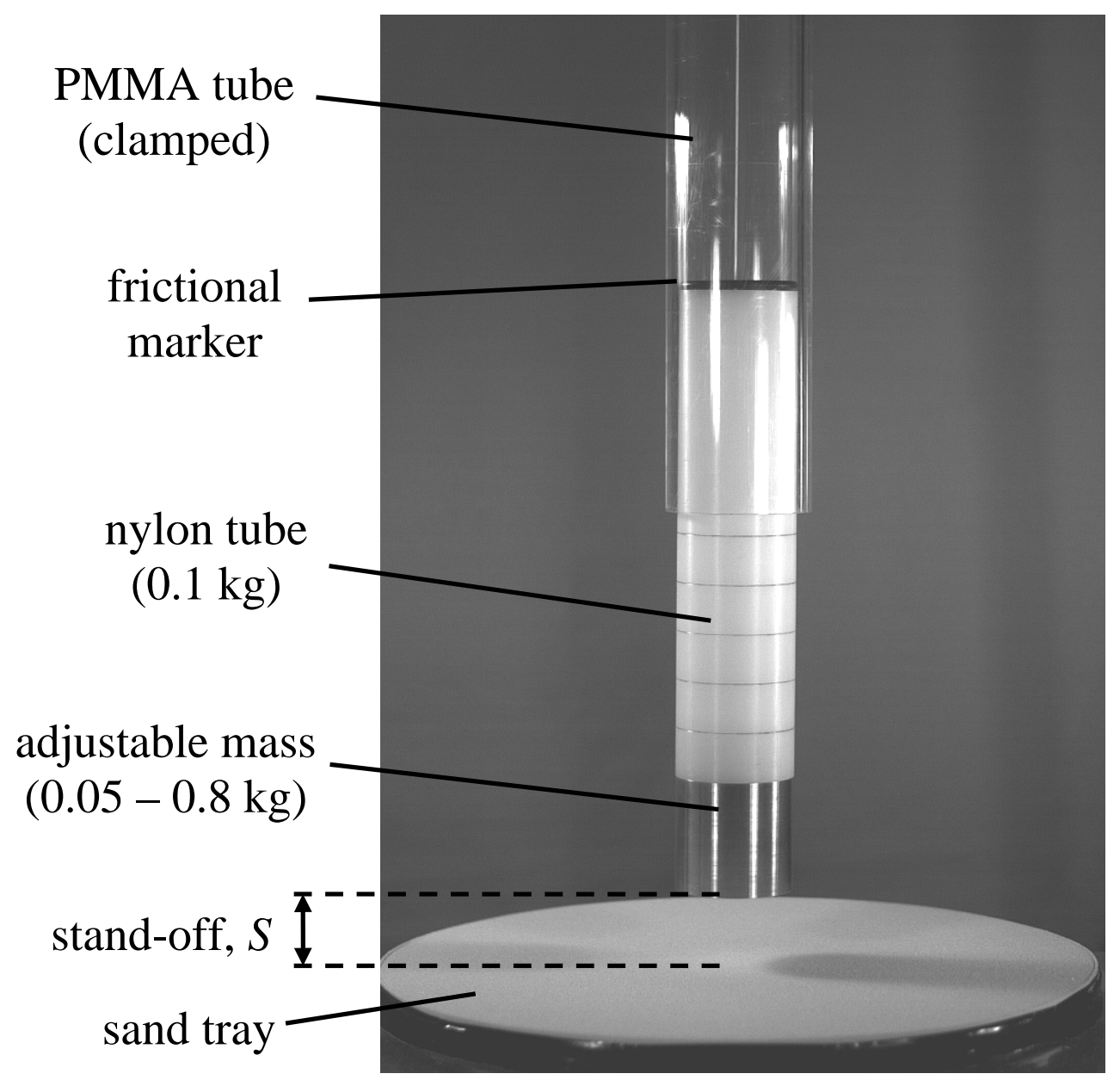

Figure 9: Photograph of the impulse pendulum apparatus. The pendulum consists of an adjustable mass of diameter $50 \mathrm{~mm}$, supported by a nylon tube, which is free to slide within a transparent PMMA tube. The position of the pendulum is monitored using high speed photography. A frictional marker confirms the maximum height obtained by the pendulum. 


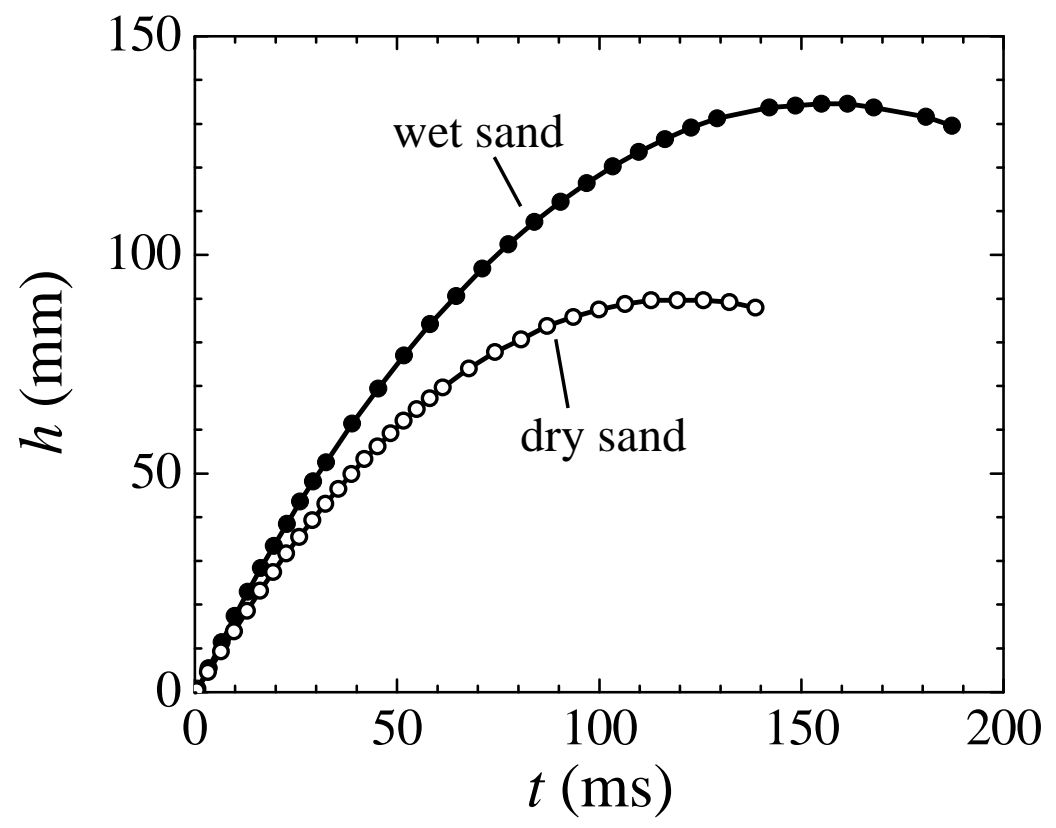

Figure 10: Vertical displacement of the impulse pendulum versus time, as measured from the high speed photography. The points denote individual measurements. Depth of burial $D=15 \mathrm{~mm}$, stand-off $S=30 \mathrm{~mm}$. The total mass of the impulse pendulum is $0.66 \mathrm{~kg}$.

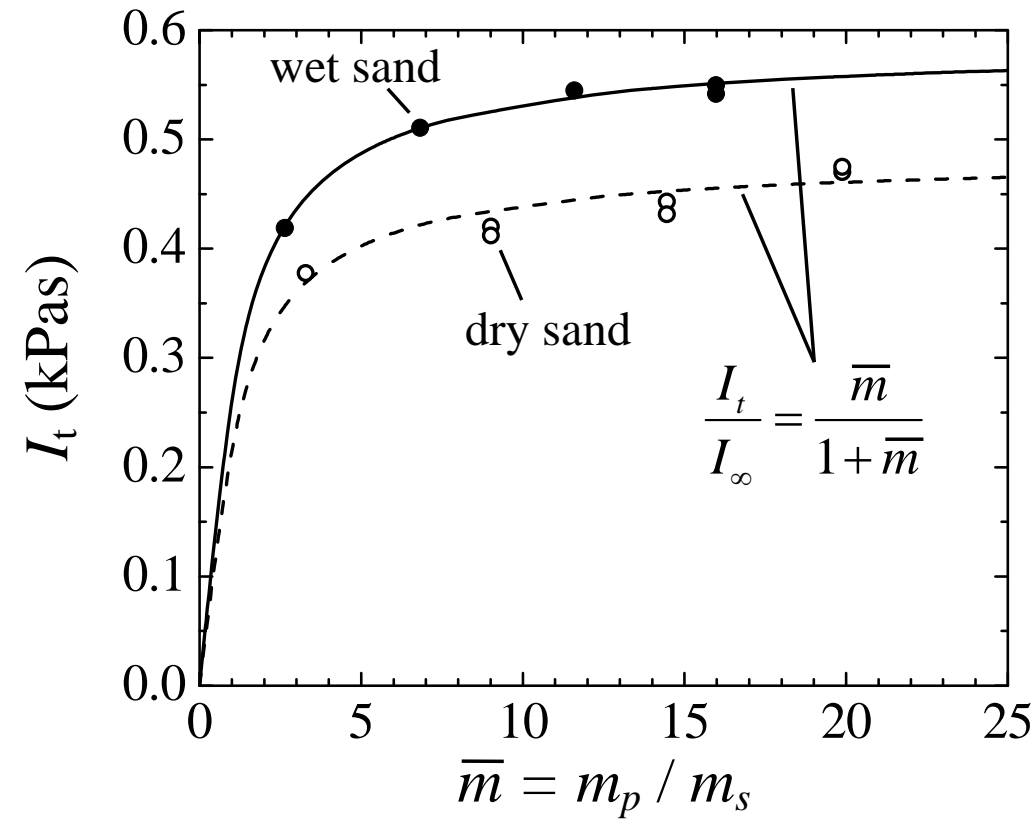

Figure 11: Variation in total transmitted impulse with the mass of the impulse pendulum. $\quad m_{p}$ and $m_{s}$ are the mass per unit area of the pendulum and sand, respectively. Points indicate individual experimental measurements. Depth of burial $D=15 \mathrm{~mm}$, stand-off $S=30 \mathrm{~mm}$. 